\title{
Cancer-associated 2-oxoglutarate analogues modify histone methylation by inhibiting histone lysine demethylases
}

\author{
Tuomas Laukka', Matti Myllykoski ${ }^{1}$, Ryan E. Looper² ${ }^{2}$ and Peppi Koivunen ${ }^{1}$ \\ ${ }^{1}$ Biocenter Oulu, Faculty of Biochemistry and Molecular Medicine, Oulu Center for Cell-Matrix Research, \\ University of Oulu, FIN-90014 Oulu, Finland \\ ${ }^{2}$ Department of Chemistry, University of Utah, Salt Lake City, UT 84112, USA
}

To whom correspondence should be addressed: Peppi Koivunen, University of Oulu, Faculty of Biochemistry and Molecular Medicine, Tel: +358 48294 5822, Fax: +358 8531 5037, E-mail: peppi.koivunen@oulu.fi 


\begin{abstract}
Histone lysine demethylases (KDMs) are 2-oxoglutarate-dependent dioxygenases (2-OGDDs) that regulate gene expression by altering chromatin structure. Their dysregulation has been associated with many cancers. We set out to study the catalytic and inhibitory properties of human KDM4A, KDM4B, KDM5B, KDM6A and KDM6B, aiming in particular to reveal which of these enzymes are targeted by cancer-associated 2-oxoglutarate (2-OG) analogues. We used affinity-purified insect cell-produced enzymes and synthetic peptides with trimethylated lysines as substrates for the in vitro enzyme activity assays. In addition, we treated breast cancer cell-lines with cell-permeable forms of 2-OG analogues and studied their effects on the global histone methylation state. Our data show that KDMs have substrate specificity. Among the enzymes studied, KDM5B had the highest affinity for the peptide substrate but the lowest affinity for the 2-OG and the $\mathrm{Fe}^{2+}$ cosubstrate/cofactors. R-2-hydroxyglutarate (R-2HG) was the most efficient inhibitor of KDM6A, KDM4A and KDM4B, followed by S-2HG. This finding was supported by accumulations of the histone H3K9me3 and H3K27me3 marks in cells treated with the cellpermeable forms of these compounds. KDM5B was especially resistant to inhibition by R-2HG, while citrate was the most efficient inhibitor of KDM6B. We conclude that KDM catalytic activity is susceptible to inhibition by tumorigenic 2-OG analogues and suggest that the inhibition of KDMs is involved in the disease mechanism of cancers in which these compounds accumulate, such as the isocitrate dehydrogenase mutations.
\end{abstract}

Keywords: KDM, fumarate, succinate, R-2HG, S-2HG

Abbreviations: KDM: Histone lysine demethylase; 2-OGDD: 2-oxoglutarate-dependent dioxygenase; 2OG: 2-oxoglutarate; R/S-2HG; R/S-2-hydroxyglutarate; TET: Ten-eleven translocation; HIF-P4H: hypoxia-inducible factor prolyl 4-hydroxylase; DEF: diethyl fumarate; DMS: dimethyl succinate; TFMBR/S-2HG: Trifluoromethyl benzyl-esterified R/S-2-hydroxyglutarate; IDH: isocitrate dehydrogenase; FH: fumarate hydratase; SDH: succinate dehydrogenase. 


\section{INTRODUCTION}

Epigenetic modifications of the nucleic acid and protein components of chromatin are central regulators of eukaryotic transcription. These modifications include the reversible methylation of DNA and histones and further histone modifications such as acetylation, phosphorylation and ubiquitination ${ }^{1}$ The chromatin modifications are written or erased by enzymes, with several of the erasers belonging to the family of 2oxoglutarate-dependent dioxygenases (2-OGDDs) ${ }^{1-4}$. The family contains $\sim 70$ enzymes in humans, including a large number of histone lysine demethylases (KDMs), the DNA demethylases, ten-eleven translocation (TET) enzymes, the hypoxia-inducible factor prolyl 4-hydroxylases (HIF-P4Hs, also known as EglNs and PHDs) and several enzymes required for collagen biosynthesis ${ }^{1,2,4}$.

The 2-OGDDs share a common reaction mechanism, although the substrate can vary from DNA to RNA, protein, fatty acid or other small molecules ${ }^{1,2,4}$. 2-OGDDs require $\mathrm{Fe}^{2+}$ as a cofactor and molecular oxygen and 2-oxoglutarate (2-OG, $\alpha$-ketoglutarate) as cosubstrates, and they yield $\mathrm{CO}_{2}$ and succinate. Many also need a reducing agent (such as vitamin C) to retain iron in the divalent state. By coupling the sensing of cellular oxygen and 2-OG levels with that of the iron redox status, the 2-OGDDs act as central cellular metabolic sensors.

2-OG is a central intermediate of the Krebs cycle, where it is produced by isocitrate dehydrogenase (IDH) isoenzymes 2 and 3. A 2-OG carrier mediates its efflux from mitochondria to the cytosol, where it can be used for amino acid synthesis or converted via reductive metabolism to acetyl-CoA and used for fatty acid synthesis. 2-OG can also be generated from several amino acids which can be converted to glutamate and transaminated to 2-OG. In the cytosol IDH1 provides an additional source of nonmitochondrial 2-OG.

2-OGDDs can be competitively antagonized by $2-\mathrm{OG}$ analogues ${ }^{1-4}$. These include the Krebs cycle intermediates fumarate, succinate, oxaloacetate and citrate, of which fumarate and succinate accumulate in fumarate hydratase $(\mathrm{FH})$ and succinate dehydrogenase $(\mathrm{SDH})$ mutant tumors, respectively ${ }^{5-8}$. IDH mutants that occur in certain cancers convert 2-OG to R-2-hydroxyglutarate (R-2HG), which is a 2-OG analogue oncometabolite capable of targeting 2-OGDDs ${ }^{9-13}$. Moreover, hypoxia and deficiency in the 
metabolizing enzyme has been shown to cause the accumulation of S-2-hydroxyglutarate (S-2HG), which can also target 2-OGDDs ${ }^{14,15}$.

Histone methylation, carried out by histone methyl transferases, occurs principally on the lysyl and arginyl side chains of histones $\mathrm{H} 3$ and $\mathrm{H} 4^{1}$. Up to three methyl groups can be added to a lysyl residue. Histone demethylation is carried out by histone demethylases, of which more than 20 are KDMs ${ }^{2,3}$. These are classified into two subfamilies one of which is the JmjC domain-containing KDM family that forms the largest subfamily within the 2-OGDDs. The KDMs are highly specific for the sequence surrounding the substrate lysine and the methylation status. Histone methylation can enable context-dependent activation or repression of transcription. In general, the methylation of histone H3 lysine 9 (H3K9) or H3K27, or histone H4 lysine 20 (H4K20) correlates with silent heterochromatin and transcriptional repression, whereas methylation of $\mathrm{H} 3 \mathrm{~K} 4, \mathrm{H} 3 \mathrm{~K} 36$ and $\mathrm{H} 3 \mathrm{~K} 79$ correlate with active euchromatin and enhanced transcription. The outcome of the methylation nevertheless depends on its site and extent, the presence of additional chromatin modifications and many other regulatory factors. KDMs play an important role in the epigenetic regulation of gene expression involved in development, cellular proliferation and differentiation, senescence and genomic stability ${ }^{1}$. Aberrant patterns of modification to chromatin have been associated with the onset and progression of many diseases, and recent evidence links several KDMs to the pathogenesis of a number of cancers ${ }^{1,16,17}$. Therefore many writers and erasers are pursued as small molecule targets for therapeutic benefit ${ }^{3}$.

Despite being high in number, the KDMs remain less well studied than the other 2-OGDDs. We therefore set out to determine the catalytic properties of several H3K4me3, H3K9me3 and H3K27me3 demethylating KDMs. We also studied the inhibitory properties of the physiologically or pathologically occurring 2-OG analogues on their activity. Finally, we used the cell-permeable forms of 2-OG analogues to study their effects on chromatin marks in cellulo. 


\section{RESULTS}

\section{Kinetic Analyses of KDMs Reveal Substrate Specificity}

In order to determine the catalytic properties of the enzymes, human full-length KDM4A, KDM4B, KDM5B and KDM6A were expressed in insect cells by means of baculoviruses as FLAG or His-tagged recombinant proteins and affinity-purified using anti-FLAG or Ni-NTA chromatography. The eluted fractions were run on SDS-PAGE followed by Coomassie Blue staining (Fig. 1a). Since a baculovirus coding for the full-length human KDM6B did not yield any soluble recombinant enzyme (data not shown), a baculovirus coding for the catalytic JmjC domain of KDM6B (aa 1164-1682) with a FLAG-tag was used instead to obtain this enzyme (Fig. 1a).

The catalytic activities of the KDMs were analysed by a method based on measurement of the lysine demethylation-coupled stoichiometric release of ${ }^{14} \mathrm{CO}_{2}$ from 2-oxo-[1- $\left.{ }^{14} \mathrm{C}\right]$ glutarate (Fig. 1d). Synthetic peptides of length 19-28 residues representing histone H3 and containing a trimethylated lysine were used as substrates (Table 1). All the purified KDMs were active under these conditions and their activity was dependent on time (Fig. 1b, c, Fig. S1A-E) and the substrate concentration (Fig. 2a-c, Fig.S2A-I), suggesting that the method was suitable for determining the catalytic properties of these enzymes.

Substrate affinity was determined first. KDM4A and KDM4B used H3K9me3 and H3K27me3 as

substrates but not H3K4me3 (Fig. 2a, Table 2, Fig.S2C-F), the $K_{m}$ values for these substrates being 20-60 $\mu \mathrm{M}$ (Table 2). KDM5B used H3K4me3 as a substrate with a $K_{m}$ value of $0.8 \mu \mathrm{M}$, but it did not demethylate the H3K9me3 or H3K27me3 substrates (Fig. 2b, Table 2). KDM5B also demethylated a dimethylated form of the $\mathrm{H} 3 \mathrm{~K} 4$ peptide with a $K_{m}$ value of $0.5 \mu \mathrm{M}$, which was highly similar to that for the trimethylated form (Table 2, Fig. S2B). KDM6A and KDM6B did not use H3K4me3 or H3K9me3 as substrates but only H3K27me3 (Fig. 2c, Table 2, Fig. S2G-I). Interestingly, KDM6A had a markedly higher $K_{m}$ value for the H3(21-44)K27(me3) substrate than did KDM6B (170 vs. $6 \mu$ M, Table 2, Fig. 
S2G, I). We therefore determined the $K_{m}$ value of KDM6A for a peptide H3(17-44)K27(me3) that was four residues longer and found it to be $130 \mu \mathrm{M}$ (Table 2, Fig. S2H).

To evaluate the turnover rate and substrate specificity, we calculated the $k_{c a t}$ and $k_{c a t} / K_{m}$ values for all enzymes and substrates (Table 2). The $k_{c a t}$ values were highly similar for all enzymes ranging from 0.004 to $0.04 \mathrm{~s}^{-1}$ (Table 2) and in the same range than the $0.015 \mathrm{~s}^{-1}$ reported earlier for KDM5B ${ }^{18}$. For KDM4A and KDM5B there was no difference in the $k_{\text {cat }}$ values between H3K9me3 and H3K27me3 and H3K4me3 and H3K4me2 substrates, respectively (Table 2). For KDM4B the $k_{\text {cat }}$ value for H3K9me3 was about double of that for the H3K27me3 substrate (Table 2). For KDM6A the $k_{c a t}$ for the longer H3K27me3 substrate was about six-fold higher than that for the four residues shorter peptide (Table 2). The $k_{\text {cat }} / K_{m}$ values for the enzymes varied from 0.016 to $0.00004 \mathrm{~s}^{-1} \mu \mathrm{M}^{-1}$ (Table 2). For KDM4A no difference in the specificity between $\mathrm{H} 3 \mathrm{~K} 9 \mathrm{me} 3$ and $\mathrm{H} 3 \mathrm{~K} 27 \mathrm{me} 3$ substrates were detected whereas for KDM4B H3K9me3 was a more specific substrate (Table 2). No major differences were detected in the specificity of KDM5B between the tri- and dimethylated H3K4 substrates whereas for KDM6A the longer H3K27me3 substrate was more specific than the shorter one (Table 2). Among H3K27me3 demethylating enzymes KDM6A had the highest specificity for this substrate (Table 2).

The $K_{m}$ values of the enzymes studied for the 2-OG cosubstrate were 6-50 $\mu \mathrm{M}$, KDM4B having the highest affinity and KDM6B the lowest (Fig. 2d, e, Table 3, Fig. S3). These values were similar to those

of HIF-P4H-1, FIH and TET2, which are other 2-OGDDs operating in the nucleus (Table 3) ${ }^{8,19,20}$. The $K_{m}$ values of the other enzymes studied here for iron $(<0.1 \mu \mathrm{M})$ were lower than that of KDM6B, which was $6 \mu \mathrm{M}$ (Fig. 2f, Table 3) and thus similar to that of TET2 ${ }^{21}$.

\section{KDMs Are Susceptible to Inhibition by 2-OG Analogues}

We next studied the inhibition of KDMs by the cancer-associated 2-OG analogues fumarate, succinate, R$2 \mathrm{HG}$ and $\mathrm{S}-2 \mathrm{HG}$ and the metabolic regulators citrate and oxaloacetate. All the recombinant KDM enzymes studied were susceptible to competitive inhibition by succinate, R-2HG, S-2HG, citrate and 
oxaloacetate (Fig. 3a-d, Table 4, Figs. S5-9). Fumarate was only a weak inhibitor of KDM4A and KDM6A, with $\mathrm{IC}_{50}$ values of 2300 and $3000 \mu \mathrm{M}$ (Fig. S4A, B), respectively, and did not inhibit KDM4B, KDM5B or KDM6B (Table 4). The oncometabolite R-2HG appeared to be the most potent inhibitor of KDM4A, KDM4B and KDM6A among the 2-OG analogues studied, with $\mathrm{IC}_{50}$ values of $<200 \mu \mathrm{M}$ (Fig.

3a, Table 4, Fig. S5A, D), and R-2HG was also the second most potent inhibitor of KDM6B, with an $\mathrm{IC}_{50}$ value of $350 \mu \mathrm{M}$ (Table 4, Fig. S5E). Unlike the other KDMs studied, R-2HG was only a very weak inhibitor of KDM5B, with an $\mathrm{IC}_{50}$ value of $3600 \mu \mathrm{M}$, which was also the case with its S-enantiomer (Table 4, Fig. S5C, Fig. S6C). On the other hand, S-2HG was the most efficient inhibitor of KDM6A among the 2-OG analogues studied, with an $\mathrm{IC}_{50}$ value of $180 \mu \mathrm{M}$, and the second and third most efficient inhibitor of KDM4A and KDM4B, respectively, with $\mathrm{IC}_{50}$ values of 290 and $450 \mu \mathrm{M}$ (Fig. 3b, Table 4, Fig. S6B). Succinate was the third and fourth most potent inhibitor of KDM6A and KDM6B, respectively, with $\mathrm{IC}_{50}$ values of 270 and $550 \mu \mathrm{M}$ (Fig. 3c, Table 4, Fig. S7E), while citrate was the most potent inhibitor of KDM6B, with an $\mathrm{IC}_{50}$ value of $130 \mu \mathrm{M}$ (Fig. 3d, Table 4), this being lower than the concentration of 2-OG in the assay. For the other KDMs studied, the $\mathrm{IC}_{50}$ values of citrate were 290$1600 \mu \mathrm{M}$ (Table 4, Fig. S8). It was also the most potent inhibitor of KDM5B among the 2-OG analogues studied, with an $\mathrm{IC}_{50}$ value of $800 \mu \mathrm{M}$ (Table 4, Fig. S8C). The $\mathrm{IC}_{50}$ values of all the KDMs studied for oxaloacetate were $380-900 \mu \mathrm{M}$ (Table 4, Fig. S9), which was the smallest variation among the $\mathrm{IC}_{50}$ values of the KDMs studied here for any of the 2-OG analogues.

\section{Treatment of Breast Cancer Cells with Cell-Permeable 2-OG Analogues Increases Global H3K27 and H3K9 Methylation Levels}

To evaluate the potential of the cancer-associated 2-OG analogues to inhibit KDMs in cellulo, we treated three subtypes of breast cancer cells, BT474 (ER+, HER2+), SK-BR-3 (ER-, HER2+) and MCF7 (ER+, HER2-) with cell-permeable forms of fumarate (DEF), succinate (DMS), R-2HG (TFMB-R-2HG) and S2HG (TFMB-S-2HG), the cells being exposed to increasing concentrations of these for $72 \mathrm{~h}$. DMSO- 
treated cells were used as controls. These compounds have been earlier reported to increase intracellular concentrations of fumarate, succinate, $\mathrm{R}-2 \mathrm{HG}$ and $\mathrm{S}-2 \mathrm{HG}^{8,20,22}$. Changes in global histone methylation with respect to H3K9 and H3K27, the marks demethylated by KDM4A, KDM4B, KDM6A and KDM6B, which were the most susceptible to inhibition by 2-OG analogues (Table 4), were determined by immunoblotting. HIF-1 $\alpha$ stabilization was also assessed in SK-BR-3 and MCF7 cells. Fumarate, R-2HG and S-2HG significantly increased both H3K27me3 and H3K9me3 levels in all three cell lines (Fig. 4), but the increases were more modest in the SK-BR-3 cells than in the BT474 and MCF7 cells (Fig. 4). Succinate slightly increased H3K27me3 and H3K9me3 levels in the BT474 and SK-BR-3 cells but these changes were modest by comparison with the other compounds (Fig. 4a, b). The most prominent effect of succinate was seen on H3K9me3 levels in the BT474 cells (Fig. 4a). R-2HG and S-2HG treatment showed dose-dependent increases in H3K27me3 and H3K9me3 levels in all the cell lines studied (Fig. 4). Fumarate, succinate, R-2HG and S-2HG stabilized HIF1 $\alpha$ in SK-BR-3 and MCF7 cells (Fig. S10). Altogether, these data show that increased intracellular 2-OG analogue concentrations can lead to increased H3K27 and H3K9 methylation. The differences in sensitivity observed in the different cells likely stem from differences in the endogenous expression levels of histone demethylases and methyltransferases, and the differences in the genomic background of the cells.

\section{DISCUSSION}

We report here on the catalytic and inhibitory properties of the high molecular weight full-length insect cell affinity-purified human enzymes KDM4A, KDM4B, KDM5B and KDM6A and the JmjC domain of KDM6B using the same assay detecting demethylation-coupled 2-OG decarboxylation in all cases. This setting provides a basis for comparing the results between the enzymes and for gaining an understanding of which enzymes are actually inhibited by the 2-OG-analogue oncometabolites and are causally linked to the transformed phenotype. Previous reports have concentrated on kinetic and inhibitory data for the catalytic domains of human KDM4A and KDM5B and full-length KDM5B, these data having been 
generated by methods that detect substrate demethylation by MALDI-TOF-MS or antibody-based AlphaScreen or formaldehyde dehydrogenase (FDH) assay, which detects formaldehyde generated by substrate hydroxylation and subsequent demethylation ${ }^{2,18,23-29}$. Of the available methods MALDI-TOFMS is the only direct method available to detect the demethylated product. The AlphaScreen technology relies on the product-recognizing antibodies. Therefore any unspecificity in them is a weakness of this method. Although being indirect the formaldehyde detected in the FDH assay stems directly from the hydoxylated product. This assay measures continuous formation of formaldehyde, unlike the others that measure product at end point. The substrate demethylation-coupled 2-OG decarboxylation assay used here measures ${ }^{14} \mathrm{C}$-labeled $\mathrm{CO}_{2}$ derived from the ${ }^{14} \mathrm{C}$-labeled 2-OG providing high specificity and sensitivity. As the 2-OGDDs can catalyse to a small extent uncoupled decarboxylation of 2-OG negative control reactions where the peptide substrate is omitted must be performed in parallel with ones containing the substrate. The benefit of the indirect assays compared with direct ones is that the one assay can be used for all enzymes that use the same reaction method. For example, the AlphaScreen requires a specific antibody for all products.

Our data show that the catalytic properties among the studies KDMs are highly similar, yet there are specificities among the enzymes. KDM4A and KDM4B demethylated peptides with trimethylated lysines in positions 9 and 27 in histone H3, while the other enzymes studied were specific for a single substrate. The former is in agreement with a previous observation, but our data do not suggest any difference in the affinity of the peptides representing the two demethylation sites in histone $\mathrm{H} 3$ for KDM4A, unlike the 20-fold higher $K_{m}$ value for H3K27me3 compared with H3K9me3 reported by others 2. Our $K_{m}$ value of $20 \mu \mathrm{M}$ for KDM4A for H3K9me3 is in agreement with those of 23-71 $\mu \mathrm{M}$ reported elsewhere for the catalytic domain of the enzyme ${ }^{2,24,26}$. The $K_{m}$ values for the two substrates studied for KDM4A and KDM4B were highly similar, KDM4B having a slightly lower affinity and higher specificity for the H3K27me3 peptide than for H3K9me3. The KDM4 paralogues A, B and C have also been reported to accept H3K36me3 and H3K36me 2 as substrates ${ }^{30}$. Among the enzymes studied here, KDM5B showed the highest affinity and specificity for its substrates. The $K_{m}$ value of $0.8 \mu \mathrm{M}$ for the 
trimethylated $\mathrm{H} 3 \mathrm{~K} 4$ was very similar to the values of 0.6 and $4.0 \mu \mathrm{M}$ reported by others using the FDHassay and peptides representing the same demethylation site as a substrate, but with only the catalytic domain of the enzyme as a catalyst ${ }^{27,28}$. The $K_{m}$ value for the dimethylated H3K4 substrate, $0.5 \mu \mathrm{M}$, was identical to that reported for the catalytic domain of KDM5B when detected by substrate demethylation with MALDI-TOF-MS ${ }^{18}$. However, the $K_{m}$ value reported for a 17-residue H3K4me3 peptide in the case of KDM5B when using substrate demethylation with AlphaScreen was $15 \mathrm{nM}^{29}$. Among the present enzymes, KDM6A had the lowest affinity for its H3(21-44)K27me3 substrate, the figure of $170 \mu \mathrm{M}$ being significantly different from the $6 \mu \mathrm{M}$ obtained for KDM6B. Since it has been reported on the basis of structural studies that amino acids 17-21 of histone H3 contribute to the substrate binding of KDM6A, we tested a longer peptide that included these amino acids as a substrate ${ }^{31}$ and found that its $K_{m}$ value was lower, $130 \mu \mathrm{M}$. It was nevertheless still the highest among the enzymes studied here, however, its kcat value for the longer peptide was highly similar if not identical with KDM6B. Altogether, these data suggest that the assay used, the substrate length and the catalyst length (full-length vs. catalytic domain) may affect the kinetic values obtained for KDMs. As the KDMs can also demethylate dimethylated lysines, and in some cases even monomethylated ones, the product of an assay using a trimethylated peptide as a substrate may serve as a substrate for a second catalytic reaction ${ }^{2,30}$. Also, the affinities for differently methylated substrates may vary, adding further complexity to the interpretation of the results.

The affinities for 2-OG and iron were highly similar for all the KDMs studied except for KDM6B, which had a $>3$-fold higher $K_{m}$ value for 2-OG and a $>60$-fold higher value for $\mathrm{Fe}^{2+}$ than any other enzyme. Such findings would suggest that when these reactants become limiting, KDM6B activity is the first to decline. Among the nuclear 2-OGDDs, the kinetics of KDM6B resembled those of TET2, whereas HIF-P4H-1 and FIH possess values that are more similar to those for the other KDMs (Table 3). While affinities of the KDMs for iron have not been reported before, our 2-OG $K_{m}$ values for KDM4A and KDM5B are in agreement with the figures of 3-15 $\mu \mathrm{M}$ reported by others for the catalytic domains of the corresponding enzymes obtained by different methods ${ }^{18,24,27}$. Intracellular 2-OG levels are estimated to 
be in the low millimolar range ${ }^{23}$, i.e. higher than the reported $K_{m}$ values of the KDMs. The concentration of 2-OG in the nucleus is not known, however, and the simultaneous presence of 2-OG and 2-OG analogues in cellulo probably alters the in vivo $K_{m}$ values of enzymes relative to the in vitro values, rendering them higher, and therefore 2-OG levels may under certain conditions be limiting for the full activity of KDMs. Iron deficiency has been shown to stabilize HIF1 ${ }^{32}$, and as the KDM6B $K_{m}$ value of 6 $\mu \mathrm{M}$ for iron was much larger than that of the HIF-P4Hs ${ }^{19}$ that regulate HIF stability, this could be expected to be of some physiological relevance.

Somatic mutations in IDH1 and IDH2 identified in gliomas, acute myeloid leukaemia, chondrosarcoma and cholangiosarcoma, and less commonly in bladder cancer, for example, lead to the accumulation of R-2HG at millimolar levels ${ }^{9-11,33-35}$. Among the 2-OG analogues studied here, R-2HG was the most potent antagonist of KDM4A, KDM4B, KDM6A and KDM6B, suggesting that the oncogenesis promoted by R-2HG involved inhibition of histone demethylation and especially accumulation of $\mathrm{H} 3 \mathrm{~K} 27 \mathrm{me} 3$ and $\mathrm{H} 3 \mathrm{~K} 9 \mathrm{me} 3$. This is in agreement with the reported accumulation of these marks in IDH1/2 mutant glial tumors ${ }^{36}$. Treatment of breast cancer cells with cell-permeable R-2HG resulted in concentration dependent-accumulation of the $\mathrm{H} 3 \mathrm{~K} 27 \mathrm{me} 3$ and $\mathrm{H} 3 \mathrm{~K} 9 \mathrm{me} 3$ marks, suggesting that R-2HG can also efficiently target the enzymes modifying these markers in cellulo. R-2HG was a more potent or equally potent inhibitor of the KDMs studied here than was S-2HG for which levels up to $300 \mu \mathrm{M}$ haven been reported in hypoxic mammalian cells ${ }^{37}$ and $750 \mu \mathrm{M}$ in S-2HG dehydrogenase mutant patients $^{38}$. This is contrary to our earlier report for other 2-OGDDs such as FIH, TET1/2, collagen prolyl 4-hydroxylase and HIF-P4Hs, for which S-2HG was always a more efficient inhibitor than R-2HG ${ }^{13}$. For the HIF-P4Hs, R-2HG is actually not an inhibitor at all but can act as a weak alternative cosubstrate instead of 2-OG ${ }^{13}$. These data suggest potential structural differences in the binding pocket for 2-OG or its competitive analogues between KDMs and other 2-OGDDs.

Succinate, which can accumulate up to millimolar levels in SDH mutant paragangliomas ${ }^{5,7}$, was a moderately potent inhibitor of KDM6A and KDM6B, which is in agreement with the reported 
accumulation of the H3K27me3 mark in $S D H$ mutant paragangliomas and pheochromocytomas ${ }^{39}$. Treatment of breast cancer cells with cell-permeable succinate nevertheless only resulted in minor increases in the $\mathrm{H} 3 \mathrm{~K} 27 \mathrm{me} 3$ marks, a higher accumulation of $\mathrm{H} 3 \mathrm{~K} 9 \mathrm{me} 3$ being detected in one cell line. These observations may suggest cell-type specific differences in the epigenetic marks mediated by succinate. Among the 2-OG analogues studied here, fumarate, which accumulates up to millimolar levels in FH-mutant renal cancers in particular ${ }^{6,7}$, was the poorest inhibitor of the KDMs with $\mathrm{IC}_{50}$ values of $\geq$ $2.3 \mathrm{mM}$. Despite the high $\mathrm{IC}_{50}$ values, cell-permeable fumarate increased the accumulation of the H3K27me3 and H3K9me3 marks in the breast cancer cells, in agreement with data reported elsewhere ${ }^{25}$. Altogether, these data suggest that fumarate may mediate its effects in these histone marks indirectly and in a manner independent of KDM inhibition.

Surprisingly, citrate, a central regulator of lipid and glucose metabolism, appeared to be a very potent inhibitor of KDM6B and a moderate inhibitor of KDM6A. Elevated plasma citrate levels up to 160 $\mu \mathrm{M}$ have been reported associated e.g. with non-alcohol fatty liver disease ${ }^{40}$. Interestingly, KDM6A activation and $\mathrm{H} 3 \mathrm{~K} 27 \mathrm{me} 3$ demethylation have been associated with brown adipocyte thermogenetic programming, which is a mechanism by which rodents combat obesity ${ }^{41}$. Moreover, ATP-citrate lyase, an enzyme that converts citrate to acetyl-CoA, is required for another modification, histone acetylation ${ }^{42}$. It has also been reported recently that chronic exposure of the heart to R-2HG, which causes contractile dysfunction, contributed to increased intracellular citrate concentration and modifications to the cardiac epigenome ${ }^{43}$. Oxaloacetate, a central regulator of several key biosynthetic pathways, such as gluconeogenesis and the synthesis of several essential amino acids, was a moderate inhibitor of KDM4A, KDM6A and $\mathrm{KDM} 6 \mathrm{~B}$ and its $\mathrm{IC}_{50}$ values did not exceed the millimolar level for any of the KDMs studied. Altogether, these data may provide an understanding of the mechanisms by which environmental factors such as diet can influence epigenetic regulation.

Although genetic alterations in KDMs have been identified in various human cancers, the precise role of KDMs in tumorigenesis remains unclear ${ }^{1,16,17}$. In some cancers the alterations lead to downregulation of the corresponding enzyme and in others to upregulation. Of the KDMs studied here, 
inactivating mutations have mainly been reported for KDM6A in lung, liver, esophageal, renal and bladder cancer, multiple myeloma and leukaemia, while KDM5B expression has been reported to be amplified in prostate, breast and bladder cancer ${ }^{44}$. Our inhibitory data support these disease mechanisms in that the catalytic activity of KDM6A, unlike that of KDM5B, was significantly more susceptible to inhibition by 2-OG-analogue oncometabolites. The tumor suppressor role of KDM6A has been further supported by its ability to prevent epithelial to mesenchymal transition in breast cancer stem cells ${ }^{45}$. For KDM4A both activating and inactivating mutations have been reported, in breast cancer and bladder cancer, respectively, while for KDM4B and KDM6B only activating mutations have been found ${ }^{44}$. The association of upregulation of KDM4A, KDM4B, KDM5B and KDM6B with certain cancers has made them targets for developing inhibitors to treat these diseases ${ }^{3}$. Our present data shed light on the mechanism behind the increased histone methylation levels observed in many cancers that involve FH, SDH and IDH mutations, but they also support the concept that it should be possible to develop specific inhibitors for the various KDMs, as they possess catalytic properties that vary from one another and are distinct from those of other 2-OGDD.

\section{MATERIALS AND METHODS}

\section{Expression and Purification of Recombinant Enzymes}

The baculoviruses for human full-length KDM5B and KDM6A with C-terminal FLAG tags were a gift from Dr. Qin Yan. cDNA clones for human full-length KDM4A and KDM4B in the pReceiver-I01 plasmid were a gift from Dr. Susanne Schlisio, and the corresponding baculoviruses with N-terminal Histags were generated. The baculoviral construct for human KDM6B 1164-1682 (coding for the catalytic JmjC domain) was amplified by PCR using the corresponding cDNA as a template. The C-terminal FLAG tag was generated by PCR and the constructs were cloned into the pVL1393 backbone. The corresponding baculovirus was generated using the BacMagic-3 DNA kit (Novagen). The baculoviruses were used to express recombinant proteins in Sf9 insect cells in TNM-FH media supplemented with $10 \%$ fetal bovine serum. The cells were infected with the respective baculoviruses and harvested $72 \mathrm{~h}$ after 
infection, washed with PBS, and homogenized in a buffer containing $10 \mathrm{mM}$ Tris- $\mathrm{HCl}(\mathrm{pH} 7.8), 150 \mathrm{mM}$ $\mathrm{NaCl}, \mu \mathrm{mM}$ glycine, $5 \mu \mathrm{M} \mathrm{FeSO}_{4}, 0.1 \%$ Triton X-100 and EDTA-free protease inhibitor (Roche).

Soluble fractions of the FLAG-tagged enzymes were purified with an anti-FLAG M2 affinity gel (Sigma). For His-tagged KDM4A and KDM4B, the soluble fractions were affinity purified using Ni-NTA agarose (Qiagen) and eluted with imidazole, which was then removed with PD-10 columns (Sigma). The fractions collected were analysed using $8 \%$ SDS-PAGE under reducing conditions followed by Coomassie Blue staining.

\section{Kinetic Activity Assays}

The catalytic activities of the KDM enzymes were determined using an assay in which the stoichiometric lysine demethylation-coupled decarboxylation of 2-OG to $\mathrm{CO}_{2}$ was detected. The assay was modified from one used to determine the catalytic activities of several 2-OGDDs ${ }^{21,46-48}$. The reactions were carried out in a final volume of $50 \mu \mathrm{l}$ and contained $50 \mathrm{mM}$ Tris- $\mathrm{HCl}(\mathrm{pH} 7.8), 2 \mathrm{mg} / \mathrm{ml}$ bovine serum albumin (Roche), $60 \mu \mathrm{g} / \mathrm{ml}$ catalase (Sigma), $0.1 \mathrm{mM}$ dithiothreitol, $2 \mathrm{mM}$ sodium ascorbate, $50 \mu \mathrm{M}$ iron(II) sulphate, $10 \%$ (v/v) dimethyl sulphoxide (DMSO), 2-oxo[1- $\left.{ }^{14} \mathrm{C}\right]$ glutarate (Perkin-Elmer) and peptide substrates representing histone H3(1-21)K4me3 (Anaspec), H3(1-21)K4me2 (Anaspec), H3(1-19)K9me3, H3(21-44)K27(me3) (Innovagen) and H3(17-44)K27(me3) (Innovagen) and 0.2-1.5 $\mu \mathrm{M}$ of the respective enzyme. $K_{m}$ values for the substrates, 2-OG and $\mathrm{Fe}^{2+}$ were determined by varying the concentration of the component in question while keeping the concentrations of the others saturating and constant. In the $\mathrm{IC}_{50}$ determinations a four-fold 2-OG concentration relative to the 2-OG $K_{m}$ value of the respective enzyme was used. These were $50 \mu \mathrm{M}$ for KDM4A, $20 \mu \mathrm{M}$ for KDM4B, $40 \mu \mathrm{M}$ for KDM5B, $37.5 \mu \mathrm{M}$ for KDM6A and $200 \mu \mathrm{M}$ for the KDM6B JmjC domain. Saturating concentrations of the other cofactors/cosubstrates were used in the presence of increasing concentrations of the respective 2-OG analogue in the reaction mixture. The reaction time was 15 or $20 \mathrm{~min}$ at $37^{\circ} \mathrm{C}$ in an ambient $\mathrm{O}_{2}$ concentration, after which the reactions were stopped by adding $100 \mu \mathrm{l}$ of $1 \mathrm{M}$ potassium phosphate $\mathrm{pH} 5$ 
and the amount of ${ }^{14} \mathrm{C}$-labelled $\mathrm{CO}_{2}$ generated was scintillated. $K_{m}$ and $V_{\max }$ values were determined from the Michaelis-Menten saturation curves and Lineweaver-Burk plots using Excel (Microsoft). The $k_{c a t}$ values were calculated from the $V_{\max }$ values taking in account the enzyme concentration and purity. $\mathrm{IC}_{50}$ values were determined from the saturation curves using Excel (Microsoft).

\section{Cell Culture Experiments with Cell-Permeable Fumarate, Succinate, R-2HG and S-2HG}

Diethyl fumarate (DEF) (Sigma) and dimethyl succinate (DMS) (Sigma) were purchased. Trifluoromethyl benzyl (TFMB)-esterified R-2-hydroxyglutarate (TFMB-R-2HG) and TFMB-S-2HG were synthesized as described before ${ }^{22}$. BT474 and SK-BR-3 cells were maintained in RPMI 1640 medium (Sigma). MCF7 cells were maintained in Dulbecco's minimal essential medium (Biochrom) supplemented with $10 \mu \mathrm{g} / \mathrm{ml}$ insulin. All media were supplemented with 10\% fetal bovine serum (Biowest). Cells were seeded 5-6 h prior to the initiation of the treatment, at $35-45 \%$ confluence and treated with increasing concentrations of DEF, DMS, TFMB-R-2HG or TFMB-S-2HG. The compounds were diluted in DMSO and control cells were treated with an equivalent volume of DMSO. The treatments lasted for $72 \mathrm{~h}$ and equal volumes and concentrations of the compounds were added to the cells every $24 \mathrm{~h}$.

\section{Immunoblotting}

Histone fractions of the cells were obtained by lysing them in a buffer containing $250 \mathrm{mM}$ sucrose, 60 $\mathrm{mM} \mathrm{KCl}, 15 \mathrm{mM} \mathrm{NaCl}, 15 \mathrm{mM}$ Tris (pH 7.5), $5 \mathrm{mM} \mathrm{MgCl} 2,1 \mathrm{mM} \mathrm{CaCl} 2,1 \mathrm{mM}$ DTT, $10 \mathrm{mM}$ butyric acid, $0.3 \%$ (v/v) NP-40 and protease inhibitor mixture without EDTA (Roche Applied Science). The lysed cells were centrifuged, the supernatant was discarded and the cell pellets were resuspended and incubated in $0.2 \mathrm{M} \mathrm{HCl}$ overnight. The samples were again centrifuged and the supernatant containing the histones was collected. The resulting histone fractions were analysed in SDS-PAGE under reducing concentrations followed by Western blotting with polyclonal rabbit antibodies against H3K27me3 (Millipore, 07-449), H3K9me3 (Abcam, ab8898) and total histone H3 (Abcam, ab1791). Polyclonal goat anti-rabbit immunoglobulin (Agilent) was used as a secondary antibody, followed by ECL 
immunodetection. For HIF1 $\alpha$ detection whole cell lysates were prepared by lysing the cells in $50 \mathrm{mM}$

Tris (pH 8.0), $150 \mathrm{mM} \mathrm{NaCl}$ and $0.5 \%$ NP40. The extracts were analyzed in SDS-PAGE under reducing conditions followed by Western blotting with monoclonal antibody against human HIF1 $\alpha$ (BD

Biosciences), followed by ECL immunodetection. Western blotting for $\beta$-actin (Novus, NB600-501) was used as a loading control.

\section{Acknowledgments:}

This work was supported by Academy of Finland Grants 266719 and 308009 (PK), and by grants from the S. Jusélius Foundation (PK), the Emil Aaltonen Foundation (PK and TL), the Finnish Medical Foundation (TL), the Jane and Aatos Erkko Foundation (PK) and the Finnish Cancer Organizations (PK).

We thank T. Aatsinki and E. Lehtimäki for their excellent technical assistance. The authors declare that they have no conflicts of interest regarding the contents of this paper.

\section{References}

1. Kaelin W. G.Jr, McKnight S. L. (2013) Influence of metabolism on epigenetics and disease. Cell 153, 56-69.

2. Williams S. T., Walport L. J., Hopkinson R. J., Madden S. K., Chowdhury R., Schofield C. J. \& Kawamura A. (2014) Studies on the catalytic domains of multiple JmjC oxygenases using peptide substrates. Epigenetics 9, 1596-1603.

3. McAllister T. E., England K. S., Hopkinson R. J., Brennan P. E., Kawamura A. \& Schofield C. J. (2016) Recent Progress in Histone Demethylase Inhibitors. J. Med. Chem. 59, 1308-1329.

4. Koivunen P., Laukka T. (2018) The TET enzymes. Cell Mol. Life Sci. 75, 1339-1348.

5. Selak M. A., Armour S. M., MacKenzie E. D., Boulahbel H., Watson D. G., Mansfield K. D., Pan Y., Simon M. C., Thompson C. B. \& Gottlieb E. (2005) Succinate links TCA cycle dysfunction to oncogenesis by inhibiting HIF-alpha prolyl hydroxylase. Cancer. Cell. 7, 77-85.

6. Isaacs J. S., Jung Y. J., Mole D. R., Lee S., Torres-Cabala C., Chung Y. L., Merino M., Trepel J., Zbar B., Toro J., Ratcliffe P. J., Linehan W. M. \& Neckers L. (2005) HIF overexpression correlates with biallelic loss of fumarate hydratase in renal cancer: novel role of fumarate in regulation of HIF stability. Cancer. Cell. 8, 143-153. 
7. Pollard P. J., Briere J. J., Alam N. A., Barwell J., Barclay E., Wortham N. C., Hunt T., Mitchell M., Olpin S., Moat S. J., Hargreaves I. P., Heales S. J., Chung Y. L., Griffiths J. R., Dalgleish A., McGrath J. A., Gleeson M. J., Hodgson S. V., Poulsom R., Rustin P. \& Tomlinson I. P. (2005) Accumulation of Krebs cycle intermediates and over-expression of HIF1alpha in tumours which result from germline FH and SDH mutations. Hum. Mol. Genet. 14, 2231-2239.

8. Koivunen P., Hirsilä M., Remes A. M., Hassinen I. E., Kivirikko K. I. \& Myllyharju J. (2007) Inhibition of hypoxia-inducible factor (HIF) hydroxylases by citric acid cycle intermediates: possible links between cell metabolism and stabilization of HIF. J. Biol. Chem. 282, 4524-4532.

9. Yan H., Parsons D. W., Jin G., McLendon R., Rasheed B. A., Yuan W., Kos I., Batinic-Haberle I., Jones S., Riggins G. J., Friedman H., Friedman A., Reardon D., Herndon J., Kinzler K. W., Velculescu V. E., Vogelstein B. \& Bigner D. D. (2009) IDH1 and IDH2 mutations in gliomas. $N$. Engl. J. Med. 360, 765-773.

10. Parsons D. W., Jones S., Zhang X., Lin J. C., Leary R. J., Angenendt P., Mankoo P., Carter H., Siu I. M., Gallia G. L., Olivi A., McLendon R., Rasheed B. A., Keir S., Nikolskaya T., Nikolsky Y., Busam D. A., Tekleab H., Diaz L. A.,Jr, Hartigan J., Smith D. R., Strausberg R. L., Marie S. K., Shinjo S. M., Yan H., Riggins G. J., Bigner D. D., Karchin R., Papadopoulos N., Parmigiani G., Vogelstein B., Velculescu V. E. \& Kinzler K. W. (2008) An integrated genomic analysis of human glioblastoma multiforme. Science 321, 1807-1812.

11. Dang L., White D. W., Gross S., Bennett B. D., Bittinger M. A., Driggers E. M., Fantin V. R., Jang H. G., Jin S., Keenan M. C., Marks K. M., Prins R. M., Ward P. S., Yen K. E., Liau L. M., Rabinowitz J. D., Cantley L. C., Thompson C. B., Vander Heiden M. G. \& Su S. M. (2009) Cancerassociated IDH1 mutations produce 2-hydroxyglutarate. Nature 462, 739-744.

12. Ward P. S., Patel J., Wise D. R., Abdel-Wahab O., Bennett B. D., Coller H. A., Cross J. R., Fantin V. R., Hedvat C. V., Perl A. E., Rabinowitz J. D., Carroll M., Su S. M., Sharp K. A., Levine R. L. \& Thompson C. B. (2010) The common feature of leukemia-associated IDH1 and IDH2 mutations is a neomorphic enzyme activity converting alpha-ketoglutarate to 2-hydroxyglutarate. Cancer. Cell. 17, 225-234.

13. Koivunen P., Lee S., Duncan C. G., Lopez G., Lu G., Ramkissoon S., Losman J. A., Joensuu P., Bergmann U., Gross S., Travins J., Weiss S., Looper R., Ligon K. L., Verhaak R. G., Yan H. \& Kaelin W. G.,Jr. (2012) Transformation by the (R)-enantiomer of 2-hydroxyglutarate linked to EGLN activation. Nature 483, 484-488.

14. Intlekofer A. M., Wang B., Liu H., Shah H., Carmona-Fontaine C., Rustenburg A. S., Salah S., Gunner M. R., Chodera J. D., Cross J. R. \& Thompson C. B. (2017) L-2-Hydroxyglutarate production arises from noncanonical enzyme function at acidic pH. Nat. Chem. Biol. 13, 494-500.

15. Van Schaftingen E., Rzem R. \& Veiga-da-Cunha M. (2009) L: -2-Hydroxyglutaric aciduria, a disorder of metabolite repair. J. Inherit. Metab. Dis. 32, 135-142.

16. Cloos P. A., Christensen J., Agger K. \& Helin K. (2008) Erasing the methyl mark: histone demethylases at the center of cellular differentiation and disease. Genes Dev. 22, 1115-1140.

17. Varier R. A., Timmers H. T. (2011) Histone lysine methylation and demethylation pathways in cancer. Biochim. Biophys. Acta 1815, 75-89. 
18. Tarhonskaya H., Nowak R. P., Johansson C., Szykowska A., Tumber A., Hancock R. L., Lang P., Flashman E., Oppermann U., Schofield C. J. \& Kawamura A. (2017) Studies on the Interaction of the Histone Demethylase KDM5B with Tricarboxylic Acid Cycle Intermediates. J. Mol. Biol. 429, 2895-2906.

19. Hirsilä M., Koivunen P., Xu L., Seeley T., Kivirikko K. I. \& Myllyharju J. (2005) Effect of desferrioxamine and metals on the hydroxylases in the oxygen sensing pathway. Faseb J. 19, 13081310 .

20. Koivunen P., Hirsilä M., Kivirikko K. I. \& Myllyharju J. (2006) The length of peptide substrates has a marked effect on hydroxylation by the hypoxia-inducible factor prolyl 4-hydroxylases. J. Biol. Chem. 281, 28712-28720.

21. Laukka T., Mariani C. J., Ihantola T., Cao J. Z., Hokkanen J., Kaelin W. G.,Jr, Godley L. A. \& Koivunen P. (2016) Fumarate and Succinate Regulate Expression of Hypoxia-inducible Genes via TET Enzymes. J. Biol. Chem. 291, 4256-4265.

22. Losman J. A., Looper R. E., Koivunen P., Lee S., Schneider R. K., McMahon C., Cowley G. S., Root D. E., Ebert B. L. \& Kaelin W. G.,Jr. (2013) (R)-2-hydroxyglutarate is sufficient to promote leukemogenesis and its effects are reversible. Science 339, 1621-1625.

23. Chowdhury R., Yeoh K. K., Tian Y. M., Hillringhaus L., Bagg E. A., Rose N. R., Leung I. K., Li X. S., Woon E. C., Yang M., McDonough M. A., King O. N., Clifton I. J., Klose R. J., Claridge T. D., Ratcliffe P. J., Schofield C. J. \& Kawamura A. (2011) The oncometabolite 2-hydroxyglutarate inhibits histone lysine demethylases. EMBO Rep. 12, 463-469.

24. Hancock R. L., Masson N., Dunne K., Flashman E. \& Kawamura A. (2017) The Activity of JmjC Histone Lysine Demethylase KDM4A is Highly Sensitive to Oxygen Concentrations. ACS Chem. Biol. 12, 1011-1019.

25. Xiao M., Yang H., Xu W., Ma S., Lin H., Zhu H., Liu L., Liu Y., Yang C., Xu Y., Zhao S., Ye D., Xiong Y. \& Guan K. L. (2012) Inhibition of alpha-KG-dependent histone and DNA demethylases by fumarate and succinate that are accumulated in mutations of FH and SDH tumor suppressors. Genes Dev. 26, 1326-1338.

26. Couture J. F., Collazo E., Ortiz-Tello P. A., Brunzelle J. S. \& Trievel R. C. (2007) Specificity and mechanism of JMJD2A, a trimethyllysine-specific histone demethylase. Nat. Struct. Mol. Biol. 14, 689-695.

27. Horton J. R., Liu X., Gale M., Wu L., Shanks J. R., Zhang X., Webber P. J., Bell J. S., Kales S. C., Mott B. T., Rai G., Jansen D. J., Henderson M. J., Urban D. J., Hall M. D., Simeonov A., Maloney D. J., Johns M. A., Fu H., Jadhav A., Vertino P. M., Yan Q. \& Cheng X. (2016) Structural Basis for KDM5A Histone Lysine Demethylase Inhibition by Diverse Compounds. Cell. Chem. Biol. 23, 769781.

28. Kristensen L. H., Nielsen A. L., Helgstrand C., Lees M., Cloos P., Kastrup J. S., Helin K., Olsen L. \& Gajhede M. (2012) Studies of H3K4me3 demethylation by KDM5B/Jarid1B/PLU1 reveals strong substrate recognition in vitro and identifies 2,4-pyridine-dicarboxylic acid as an in vitro and in cell inhibitor. Febs J. 279, 1905-1914. 
29. Sayegh J., Cao J., Zou M. R., Morales A., Blair L. P., Norcia M., Hoyer D., Tackett A. J., Merkel J. S. \& Yan Q. (2013) Identification of small molecule inhibitors of Jumonji AT-rich interactive domain 1B (JARID1B) histone demethylase by a sensitive high throughput screen. J. Biol. Chem. 288, 9408-9417.

30. Whetstine J. R., Nottke A., Lan F., Huarte M., Smolikov S., Chen Z., Spooner E., Li E., Zhang G., Colaiacovo M. \& Shi Y. (2006) Reversal of histone lysine trimethylation by the JMJD2 family of histone demethylases. Cell 125, 467-481.

31. Sengoku T., Yokoyama S. (2011) Structural basis for histone H3 Lys 27 demethylation by UTX/KDM6A. Genes Dev. 25, 2266-2277.

32. Peyssonnaux C., Zinkernagel A. S., Schuepbach R. A., Rankin E., Vaulont S., Haase V. H., Nizet V. \& Johnson R. S. (2007) Regulation of iron homeostasis by the hypoxia-inducible transcription factors (HIFs). J. Clin. Invest. 117, 1926-1932.

33. Ward P. S., Patel J., Wise D. R., Abdel-Wahab O., Bennett B. D., Coller H. A., Cross J. R., Fantin V. R., Hedvat C. V., Perl A. E., Rabinowitz J. D., Carroll M., Su S. M., Sharp K. A., Levine R. L. \& Thompson C. B. (2010) The common feature of leukemia-associated IDH1 and IDH2 mutations is a neomorphic enzyme activity converting alpha-ketoglutarate to 2-hydroxyglutarate. Cancer. Cell. 17, 225-234.

34. Amary M. F., Bacsi K., Maggiani F., Damato S., Halai D., Berisha F., Pollock R., O'Donnell P., Grigoriadis A., Diss T., Eskandarpour M., Presneau N., Hogendoorn P. C., Futreal A., Tirabosco R. \& Flanagan A. M. (2011) IDH1 and IDH2 mutations are frequent events in central chondrosarcoma and central and periosteal chondromas but not in other mesenchymal tumours. J. Pathol. 224, 334343.

35. Cerami E., Gao J., Dogrusoz U., Gross B. E., Sumer S. O., Aksoy B. A., Jacobsen A., Byrne C. J., Heuer M. L., Larsson E., Antipin Y., Reva B., Goldberg A. P., Sander C. \& Schultz N. (2012) The cBio cancer genomics portal: an open platform for exploring multidimensional cancer genomics data. Cancer. Discov. 2, 401-404.

36. Lu C., Ward P. S., Kapoor G. S., Rohle D., Turcan S., Abdel-Wahab O., Edwards C. R., Khanin R., Figueroa M. E., Melnick A., Wellen K. E., O'Rourke D. M., Berger S. L., Chan T. A., Levine R. L., Mellinghoff I. K. \& Thompson C. B. (2012) IDH mutation impairs histone demethylation and results in a block to cell differentiation. Nature 483, 474-478.

37. Intlekofer A. M., Dematteo R. G., Venneti S., Finley L. W., Lu C., Judkins A. R., Rustenburg A. S., Grinaway P. B., Chodera J. D., Cross J. R. \& Thompson C. B. (2015) Hypoxia Induces Production of L-2-Hydroxyglutarate. Cell. Metab. 22, 304-311.

38. Kranendijk M., Struys E. A., Salomons G. S., Van der Knaap M. S. \& Jakobs C. (2012) Progress in understanding 2-hydroxyglutaric acidurias. J. Inherit. Metab. Dis. 35, 571-587.

39. Letouze E., Martinelli C., Loriot C., Burnichon N., Abermil N., Ottolenghi C., Janin M., Menara M., Nguyen A. T., Benit P., Buffet A., Marcaillou C., Bertherat J., Amar L., Rustin P., De Reynies A., Gimenez-Roqueplo A. P. \& Favier J. (2013) SDH mutations establish a hypermethylator phenotype in paraganglioma. Cancer. Cell. 23, 739-752. 
40. van de Wier B., Balk J. M., Haenen G. R., Giamouridis D., Bakker J. A., Bast B. C., den Hartog G. J., Koek G. H. \& Bast A. (2013) Elevated citrate levels in non-alcoholic fatty liver disease: the potential of citrate to promote radical production. FEBS Lett. 587, 2461-2466.

41. Zha L., Li F., Wu R., Artinian L., Rehder V., Yu L., Liang H., Xue B. \& Shi H. (2015) The Histone Demethylase UTX Promotes Brown Adipocyte Thermogenic Program Via Coordinated Regulation of H3K27 Demethylation and Acetylation. J. Biol. Chem. 290, 25151-25163.

42. Wellen K. E., Hatzivassiliou G., Sachdeva U. M., Bui T. V., Cross J. R. \& Thompson C. B. (2009) ATP-citrate lyase links cellular metabolism to histone acetylation. Science 324, 1076-1080.

43. Karlstaedt A., Zhang X., Vitrac H., Harmancey R., Vasquez H., Wang J. H., Goodell M. A. \& Taegtmeyer H. (2016) Oncometabolite d-2-hydroxyglutarate impairs alpha-ketoglutarate dehydrogenase and contractile function in rodent heart. Proc. Natl. Acad. Sci. U. S. A. 113, 1043610441.

44. Park S. Y., Park J. W. \& Chun Y. S. (2016) Jumonji histone demethylases as emerging therapeutic targets. Pharmacol. Res. 105, 146-151.

45. Choi H. J., Park J. H., Park M., Won H. Y., Joo H. S., Lee C. H., Lee J. Y. \& Kong G. (2015) UTX inhibits EMT-induced breast CSC properties by epigenetic repression of EMT genes in cooperation with LSD1 and HDAC1. EMBO Rep. 16, 1288-1298.

46. Kivirikko K. I., Myllylä R. (1982) Posttranslational enzymes in the biosynthesis of collagen: intracellular enzymes. Methods Enzymol. 82 Pt A, 245-304.

47. Hirsilä M., Koivunen P., Gunzler V., Kivirikko K. I. \& Myllyharju J. (2003) Characterization of the human prolyl 4-hydroxylases that modify the hypoxia-inducible factor. J. Biol. Chem. 278, 3077230780 .

48. Koivunen P., Hirsilä M., Gunzler V., Kivirikko K. I. \& Myllyharju J. (2004) Catalytic properties of the asparaginyl hydroxylase (FIH) in the oxygen sensing pathway are distinct from those of its prolyl 4-hydroxylases. J. Biol. Chem. 279, 9899-9904. 


\section{FIGURE LEGENDS}

Fig. 1. Expression, purification and catalytical activity measurements of KDMs. (a) SDS-PAGE and Coomassie Blue analysis of affinity purified human recombinant KDM4A (4A), KDM4B (4B), KDM5B (5B), KDM6A (6A) and KDM6B (6B*). For KDM6B the JmjC catalytic domain was expressed. (b-c) Time course analysis of KDM4B and KDM6A. KDM4B (b) and KDM6A (c) retained a linear increase in velocity as a function of time up to $20 \mathrm{~min}$. $100 \mu \mathrm{M} \mathrm{H} 3 \mathrm{~K} 9 \mathrm{me} 3$ was used as a substrate in (b) and $200 \mu \mathrm{M}$ $\mathrm{H} 3(21-44) \mathrm{K} 27 \mathrm{me} 3$ in (c). (d) Schematic drawing of KDM catalytic reaction and analysis catalytical activity by determination of released ${ }^{14} \mathrm{CO}_{2}$ from the stoichiometric lysine demethylation-coupled decarboxylation of 2-OG to $\mathrm{CO}_{2}$.

Fig. 2. Kinetic analyses of KDMs. Michaelis-Menten curves and Lineweaver-Burk plots (inset) of KDM4A for H3(21-44)K27me3 substrate (a), KDM5B for H3K4(1-21)me3 substrate (b), KDM6A for H3(21-44)K27me3 substrate (c), KDM6A for 2-oxoglutarate (d), KDM6B* for 2-oxoglutarate (e) and KDM6B* for iron (f). *JmjC domain.

Fig. 3. KDMs are susceptible to competitive inhibition by 2-OG analogues. (a-b) $\mathrm{IC}_{50}$ curves of KDM4B and KDM4A for R-2HG and S-2HG, respectively. (c-d) $\mathrm{IC}_{50}$ curves of KDM6A and KDM6B for succinate and citrate, respectively.

Fig. 4. Treatment of cells with cell-permeable forms of fumarate, succinate, R-2HG and S-2HG results in increased global histone methylation. $\mathrm{H} 3 \mathrm{~K} 27 \mathrm{me} 3$ and $\mathrm{H} 3 \mathrm{~K} 9 \mathrm{me} 3$ levels determined by immunoblotting of BT474 (a), SK-BR-3 (b) and MCF7 (c) cells incubated with increasing concentrations of diethyl fumarate (DEF), dimethyl succinate (DMS), TFMB-R-2HG and TFMB-S-2HG for $72 \mathrm{~h}$. 
Table 1. Synthetic peptides representing histone $\mathrm{H3}$ demethylation sites used as substrates for KDMs.

\begin{tabular}{lll}
\hline Substrate & Sequence & Manufacturer \\
\hline H3K4me3(1-21) & ART-K(Me3)-QTARKSTGGKAPRKQLA-GGK(Biotin) & Anaspec \\
H3K4me2(1-21) & ART-K(Me2)-QTARKSTGGKAPRKQLA-GGK(Biotin) & Anaspec \\
H3K9me3(1-19) & MARTKQTAR-K(Me3)-STGGKAPRKQ & Innovagen \\
H3K27me3(21-44) & ATKAAR-K(Me3)-SAPATGGVKKPHRYRPG-GK(Biotin) & Innovagen, \\
H3K27me3(17-44) & RKQLATKAAR-(KMe3)-SAPATGGVKKPHRYRPG-GK(Biotin) & Innovagen \\
\hline
\end{tabular}


Table 2. $K_{m}, \boldsymbol{k}_{c a t}$ and $\boldsymbol{k}_{c a t} / K_{m}$ values of KDM4A, KDM4B, KDM5B, KDM6A and KDM6B* for methylated histone substrates.

\begin{tabular}{|c|c|c|c|c|c|c|}
\hline & & H3K4me3 & H3K4me2 & H3K9me3 & $\begin{array}{l}\mathrm{H} 3(21-44) \\
\text { K27me3 }\end{array}$ & $\begin{array}{c}\mathrm{H} 3(17-44) \\
\text { K27me3 }\end{array}$ \\
\hline \multirow[t]{3}{*}{ KDM4A } & $K_{m}(\mu M)$ & $>1000 /$ inactive & - & $20 \pm 10$ & $17 \pm 4$ & - \\
\hline & $k_{\text {cat }}\left(s^{-1}\right)$ & & & $0.005 \pm 0.001$ & $0.004 \pm 0.0007$ & \\
\hline & $k_{c a t} / K_{m}\left(s^{-1} \mu M^{-1}\right)$ & & & 0.00025 & 0.00024 & \\
\hline \multirow[t]{3}{*}{ KDM4B } & $K_{m}(\mu M)$ & $>2000 /$ inactive & - & $30 \pm 17$ & $60 \pm 20$ & - \\
\hline & $k_{\text {cat }}\left(s^{-1}\right)$ & & & $0.009 \pm 0.003$ & $0.004 \pm 0.002$ & \\
\hline & $k_{c a t} / K_{m}\left(s^{-1} \mu M^{-1}\right)$ & & & 0.0003 & 0.00007 & \\
\hline \multirow[t]{3}{*}{ KDM5B } & $K_{m}(\mu M)$ & $0.8 \pm 0.3$ & $0.5 \pm 0.3$ & $>2000 /$ inactive & $>2000 /$ inactive & - \\
\hline & $k_{c a t}\left(s^{-1}\right)$ & $0.009 \pm 0.003$ & $0.008 \pm 0.003$ & & & \\
\hline & $k_{c a t} / K_{m}\left(s^{-1} \mu M^{-1}\right)$ & 0.011 & 0.016 & & & \\
\hline \multirow[t]{3}{*}{ KDM6A } & $K_{m}(\mu M)$ & $>2000 /$ inactive & - & $>2000 /$ inactive & $170 \pm 40$ & $130 \pm 30$ \\
\hline & $k_{c a t}\left(s^{-1}\right)$ & & & & $0.007 \pm 0.001$ & $0.04 \pm 0.01$ \\
\hline & $k_{c a t} / K_{m}\left(s^{-1} \mu M^{-1}\right)$ & & & & 0.00004 & 0.003 \\
\hline \multirow[t]{3}{*}{ KDM6B* } & $K_{m}(\mu M)$ & $>2000 /$ inactive & - & $>2000 /$ inactive & $6 \pm 3$ & - \\
\hline & $k_{\text {cat }}\left(s^{-1}\right)$ & & & & $0.015 \pm 0.0005$ & \\
\hline & $k_{c a t} / K_{m}\left(s^{-1} \mu M^{-1}\right)$ & & & & 0.0025 & \\
\hline
\end{tabular}

Values are means \pm S.D. of at least 3 independent assays.

*JmjC domain 
Table 3. $K_{m}$ values of KDM4A, KDM4B, KDM5B, KDM6A and KDM6B* for 2-oxoglutarate and $\mathrm{Fe}^{2+}$ by comparison with values of selected nuclear resident 2-oxoglutarate-dependent dioxygenases. References indicate values not determined here.

\begin{tabular}{lcc}
\hline & $2-\mathrm{OG}$ & $\mathrm{Fe}^{2+}$ \\
& $\mu M$ & \\
\hline KDM4A & $15 \pm 9$ & $<0.1$ \\
KDM4B & $6 \pm 3$ & $<0.1$ \\
KDM5B & $10 \pm 2$ & $<0.1$ \\
KDM6A & $8 \pm 4$ & $<0.1$ \\
KDM6B $*$ & $50 \pm 20$ & $6 \pm 2$ \\
HIF-P4H-1 & $2 \pm 0.4^{8}$ & $0.03 \pm 0.002^{18}$ \\
FIH & $25 \pm 3^{46}$ & $0.5 \pm 0.2^{46}$ \\
TET2 & $55 \pm 20^{20}$ & $4.8 \pm 4^{20}$ \\
\hline
\end{tabular}

Values are means \pm S.D. of at least 3 independent assays.

*JmjC domain 
Table 4. $\mathrm{IC}_{50}$ values of succinate, fumarate, $(R)$-, $(S)$-2-hydroxyglutarate, citrate and oxaloacetate

for KDM4A, KDM4B, KDM5B, KDM6A and KDM6B* by comparison with values of selected nuclear resident 2-oxoglutarate-dependent dioxygenases. References indicate values not determined here.

\begin{tabular}{lcccccc}
\hline & Fumarate & Succinate & $\begin{array}{c}\text { R-2HG } \\
\mu M\end{array}$ & S-2HG & Citrate & Oxaloacetate \\
\hline KDM4A & $2300 \pm 200$ & $800 \pm 150$ & $160 \pm 10$ & $290 \pm 20$ & $800 \pm 250$ & $300 \pm 50$ \\
KDM4B & $>5000$ & $2300 \pm 900$ & $150 \pm 30$ & $450 \pm 130$ & $1600 \pm 200$ & $700 \pm 50$ \\
KDM5B & $>5000$ & $1400 \pm 500$ & $3600 \pm 1400$ & $1600 \pm 200$ & $800 \pm 300$ & $900 \pm 100$ \\
KDM6A & $3000 \pm 1000$ & $270 \pm 40$ & $180 \pm 30$ & $180 \pm 60$ & $290 \pm 40$ & $440 \pm 50$ \\
KDM6B & $>5000$ & $550 \pm 80$ & $350 \pm 100$ & $750 \pm 250$ & $130 \pm 10$ & $380 \pm 80$ \\
HIF-P4H-1 & $120 \pm 10^{8}$ & $830 \pm 540^{8}$ & not an inhibitor ${ }^{13}$ & $630^{13, \#}$ & $6300 \pm 1300^{8}$ & $1000 \pm 420^{8}$ \\
FIH & $>10000^{8}$ & $>10000^{8}$ & $1100-1500^{13,21}$ & $190-300^{13,21}$ & $850 \pm 190^{8}$ & $1400 \pm 370^{8}$ \\
TET2 & $400 \pm 70^{20}$ & $570 \pm 190^{20}$ & $5000^{13}$ & $1600^{13}$ & $>5000^{20}$ & not determined \\
\hline
\end{tabular}

Values are means \pm S.D. of at least 3 independent assays.

$*$ JmjC domain.

${ }^{\#} K_{i}$ value 


\section{Supplemental data}

Cancer-associated 2-oxoglutarate analogues modify histone methylation by inhibiting histone lysine demethylases

Tuomas Laukka, Matti Myllykoski, Ryan E. Looper and Peppi Koivunen
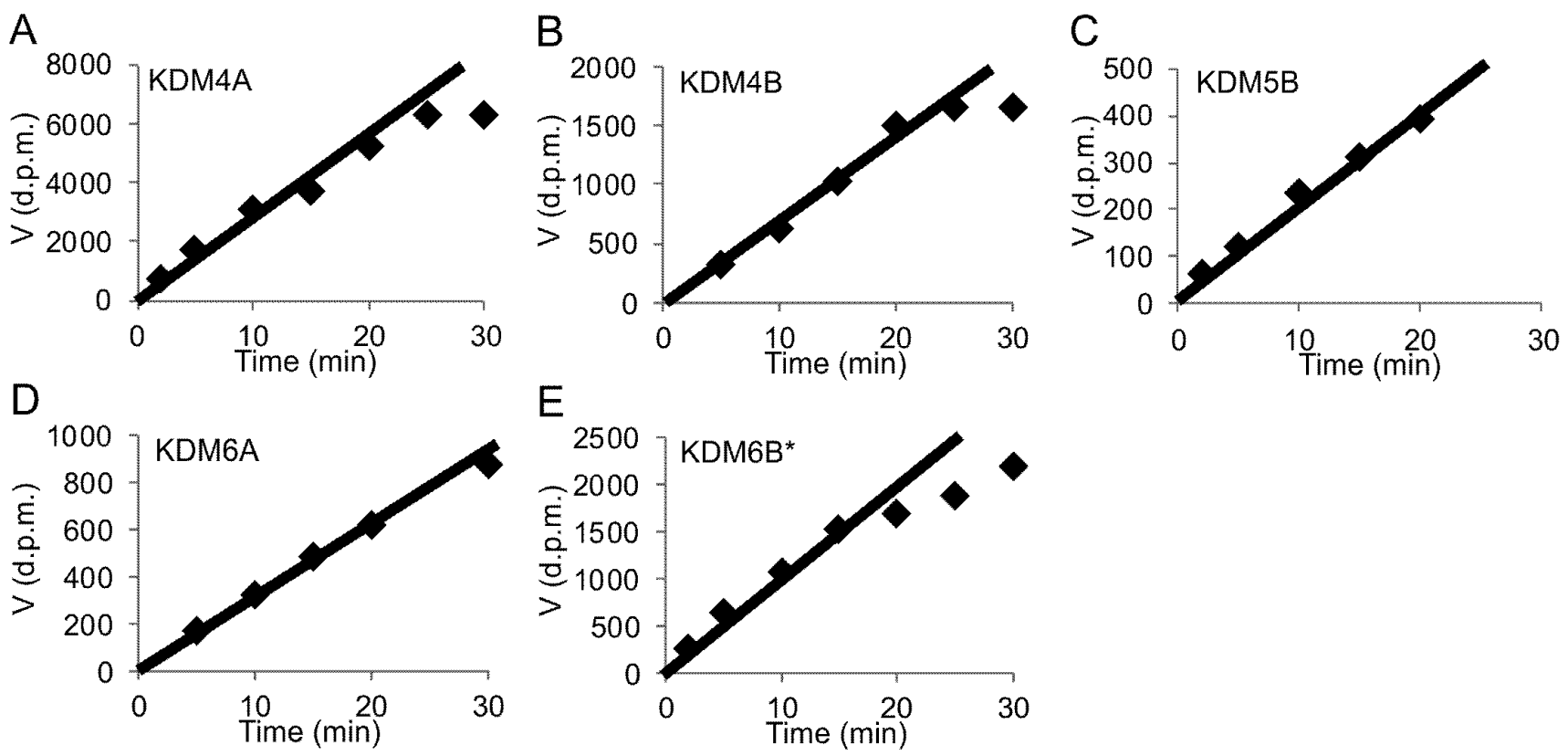

Supp. Fig. 1. Time course analysis of KDM4A, KDM4B, KDM5B, KDM6A and KDM6B. All enzymes, except KDM6B*, retained a linear increase in velocity as a function of time for at least 20 min (a-d). KDM6B* retained a linear increase in velocity as a function of time for $15 \mathrm{~min}(\mathrm{e})$. Reaction times for enzyme kinetic activity assays were adjusted accordingly. 100 and $500 \mu \mathrm{M} \mathrm{H} 3 \mathrm{~K} 9 \mathrm{me} 3$ was used as a substrate in (a) and (b), respectively, $20 \mu \mathrm{M} \mathrm{H} 3 \mathrm{~K} 4 \mathrm{me} 3$ in (c) and 1000 and $100 \mu \mathrm{M} \mathrm{H} 3(21-44) \mathrm{K} 27 \mathrm{me} 3$ in (d) and (e), respectively. 
A
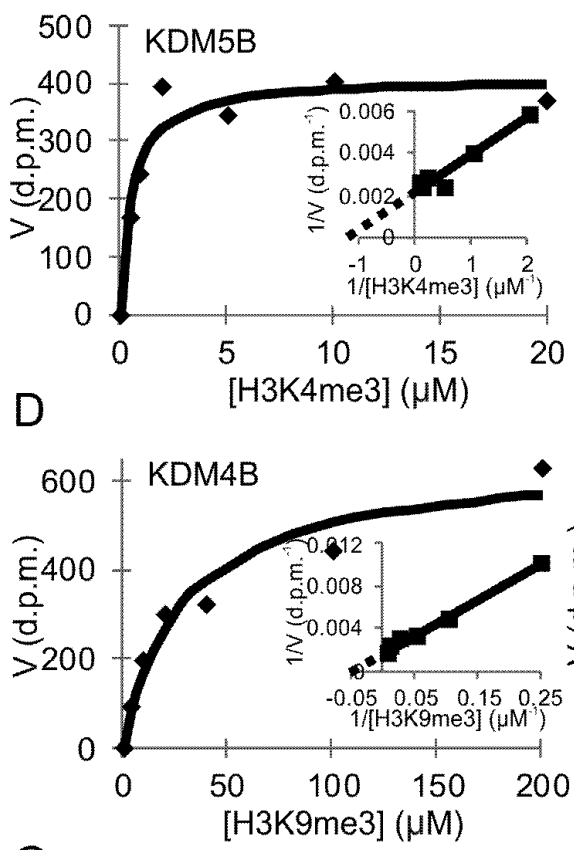

G

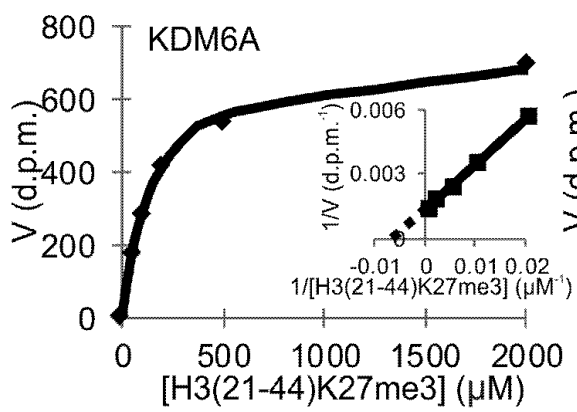

B

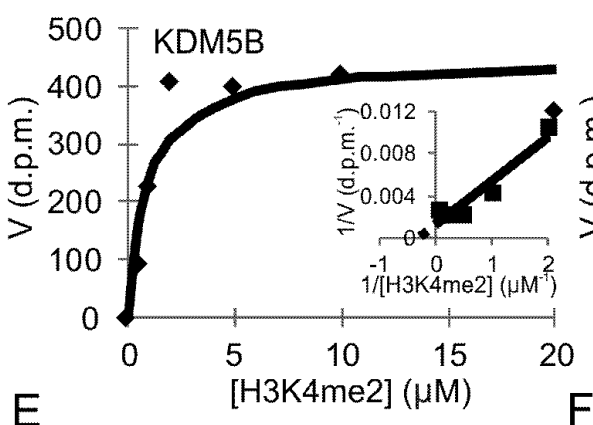

C

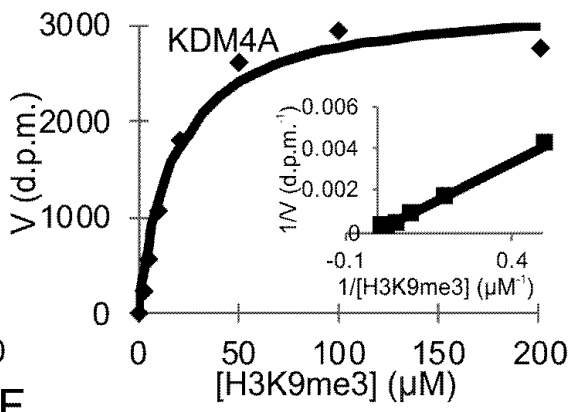

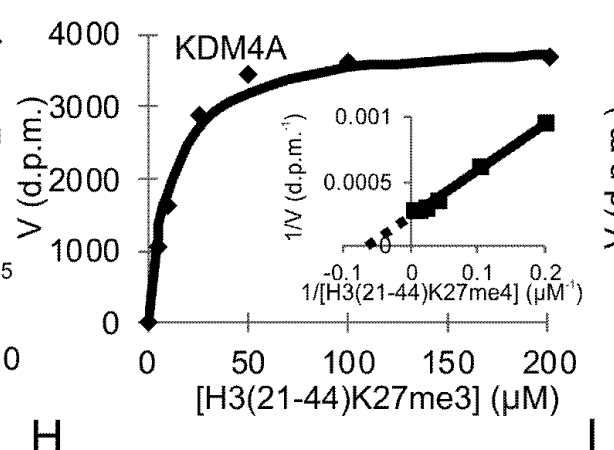

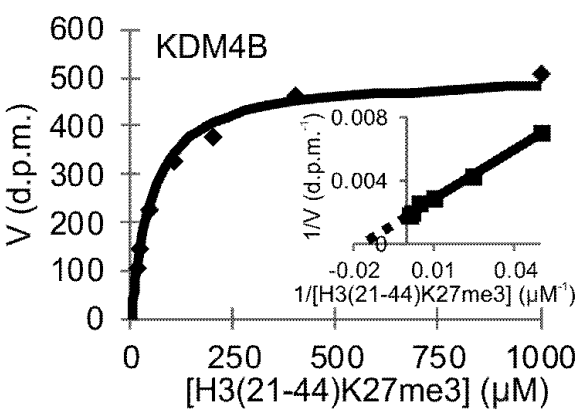

1500 T KDM6A

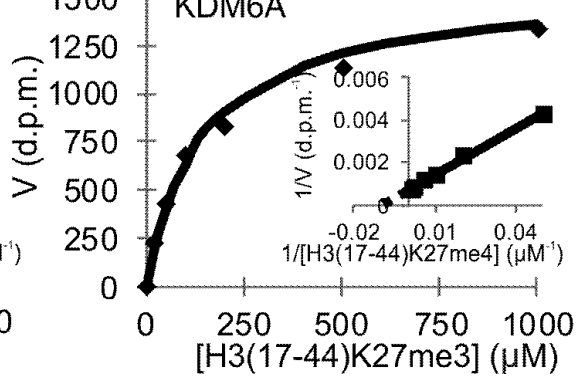

Supp. Fig. 2. Kinetic analyses of KDMs for different substrates. Michaelis-Menten curves and Lineweaver-Burk plots (inset) of KDM5B for H3(1-21)K4me3 (a) and H3(1-21)K4me2 substrate (b), KDM4A and KDM4B for H3K9(1-19)me3 (c-d) and H3(21-44)K27me3 substrate (e-f), KDM6A for H3(21-44)K27me3 (g) and H3(1744)K27me3 substrate (h), and KDM6B* for H3(21-44)K27me3 substrate (i). *JmjC domain. 

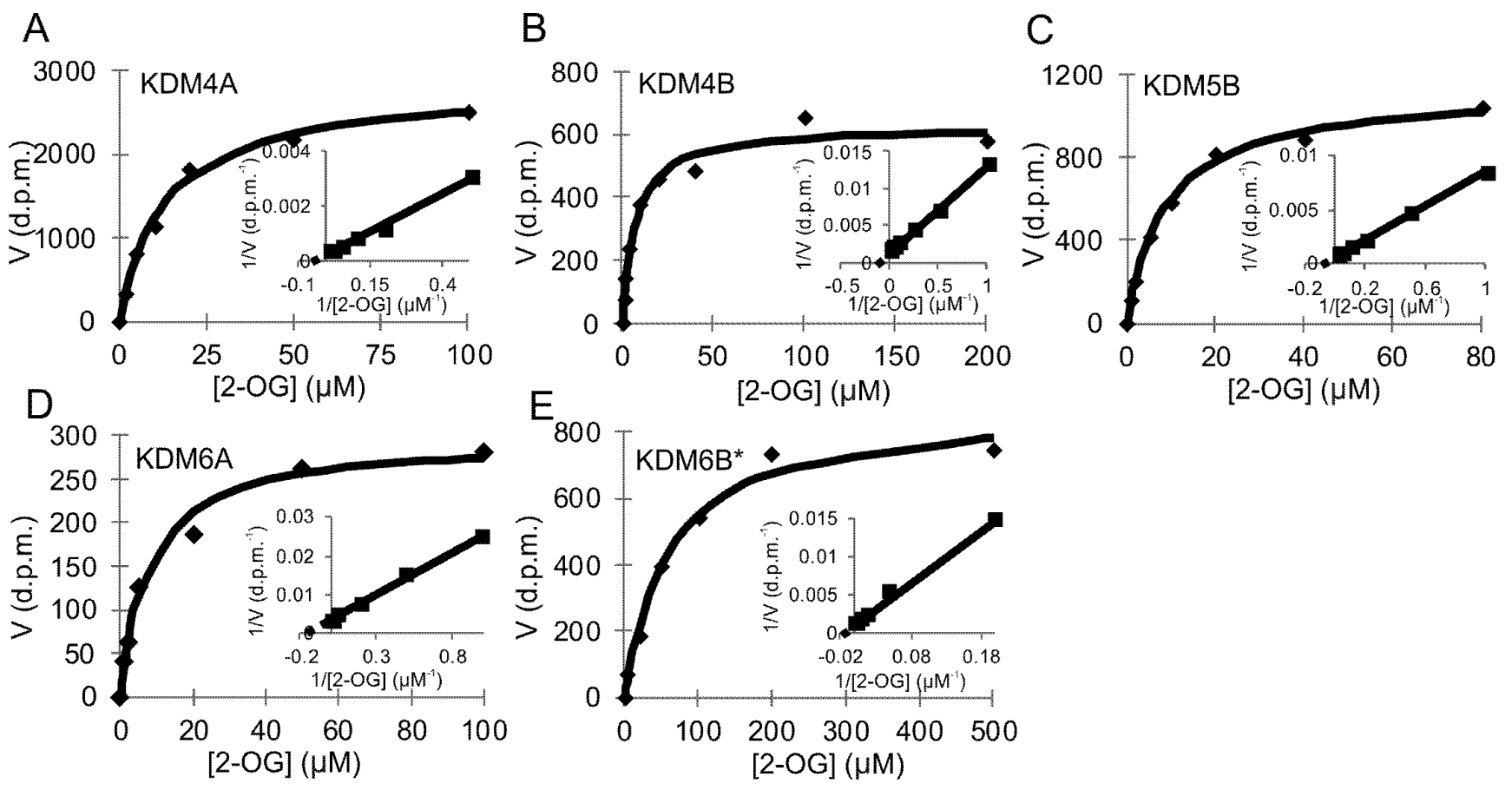

Supp. Fig. 3. Kinetic analyses of KDMs for 2-OG. Michaelis-Menten curves and Lineweaver-Burk plots (inset) of KDM4A (a), KDM4B (b), KDM5B (c), KDM6A (d) and KDM6B* (e) for 2-OG. *JmjC domain. 

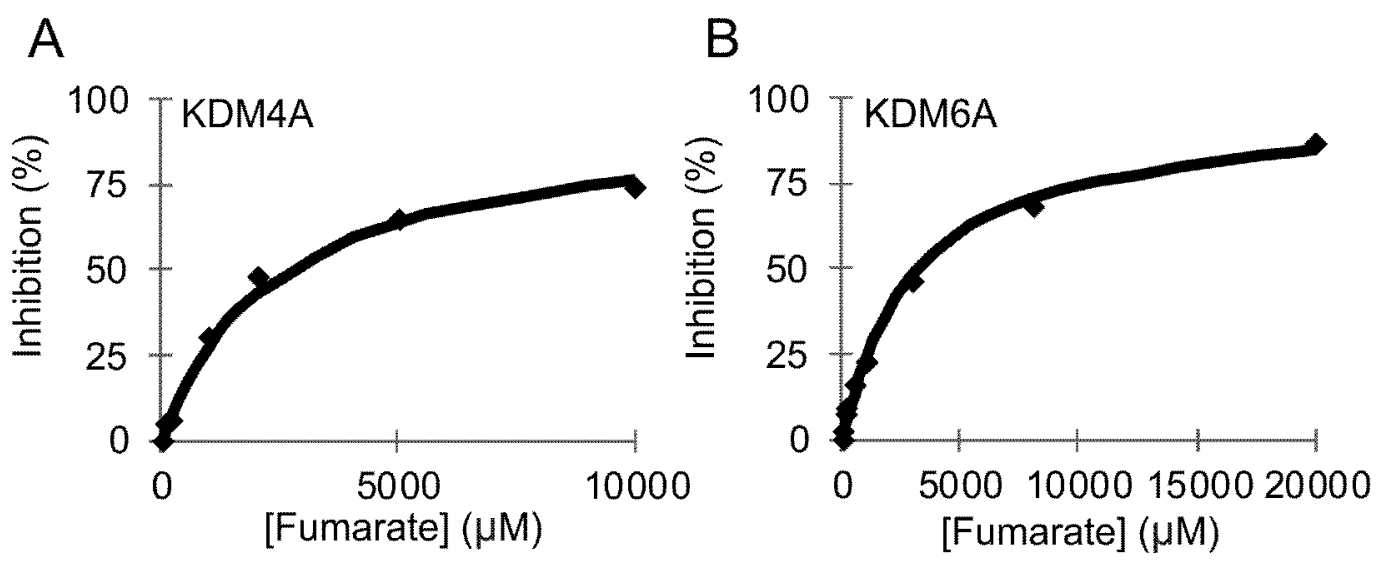

Supp. Fig. 4. KDM4A and KDM6A are susceptible to competitive inhibition by fumarate. $\mathrm{IC}_{50}$ curves of KDM4A (a) and KDM6A (b) for fumarate. 


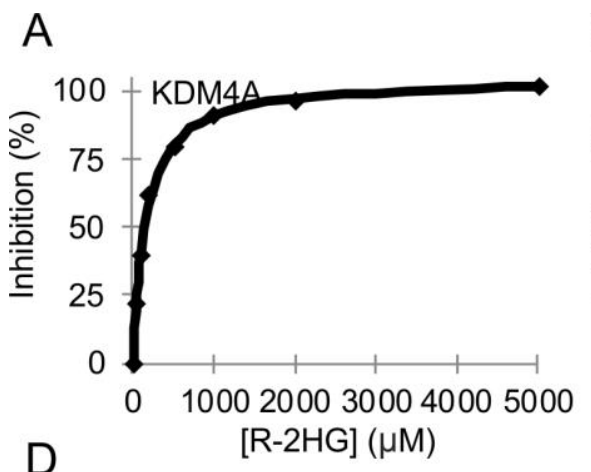

B

C
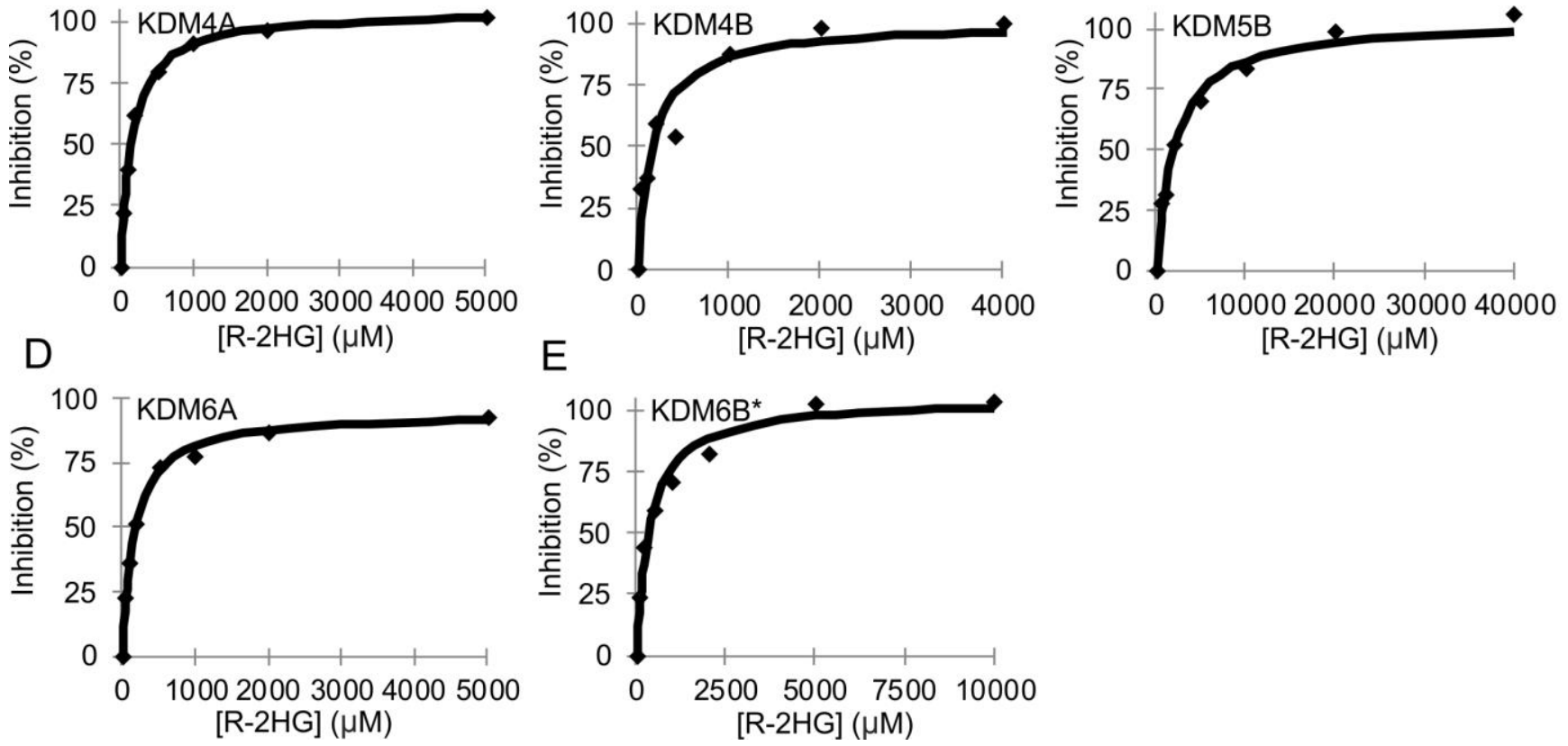

Supp. Fig. 5. KDMs are susceptible to competitive inhibition by R-2HG. IC $5_{50}$ curves of KDM4A (a), KDM4B (b), KDM5B (c), KDM6A (d) and KDM6B* (e) for R-2HG. *JmjC domain. 

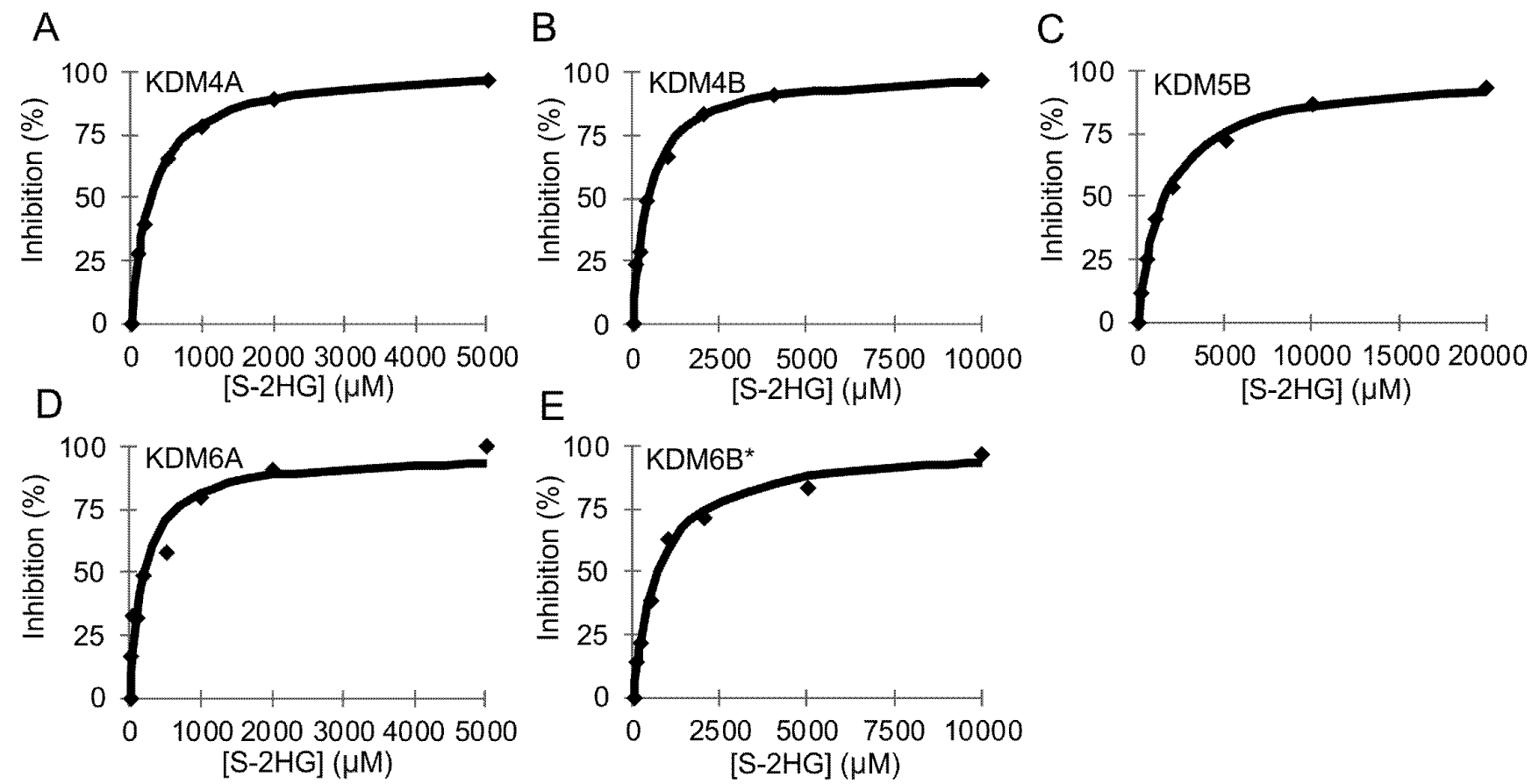

Supp. Fig. 6. KDMs are susceptible to competitive inhibition by S-2HG. IC 50 curves of KDM4A (a), KDM4B (b), KDM5B (c), KDM6A (d) and KDM6B* (e) for S-2HG. *JmjC domain. 

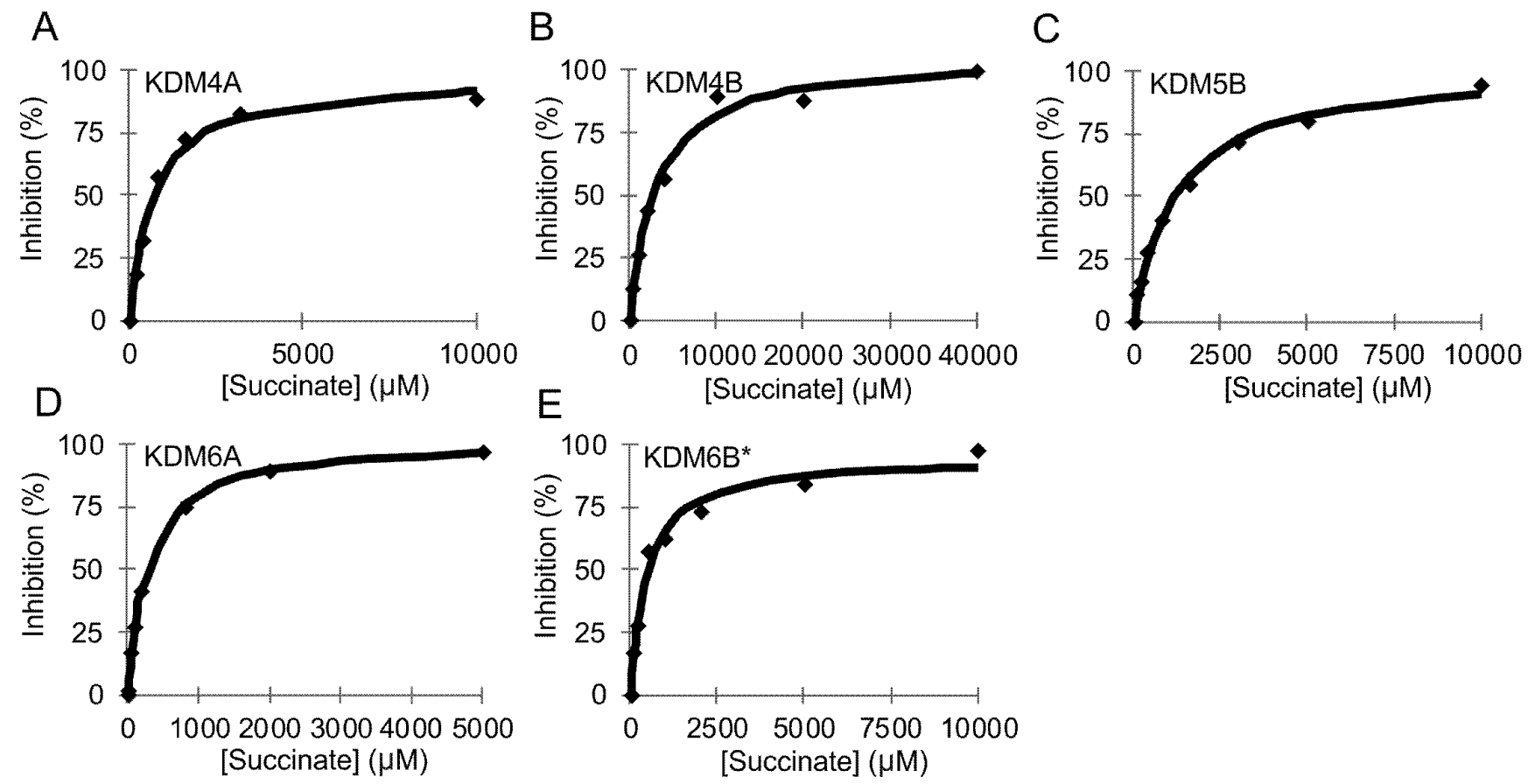

Supp. Fig. 7. KDMs are susceptible to competitive inhibition by succinate. IC $_{50}$ curves of KDM4A (a), KDM4B (b), KDM5B (c), KDM6A (d) and KDM6B* (e) for succinate. *JmjC domain. 

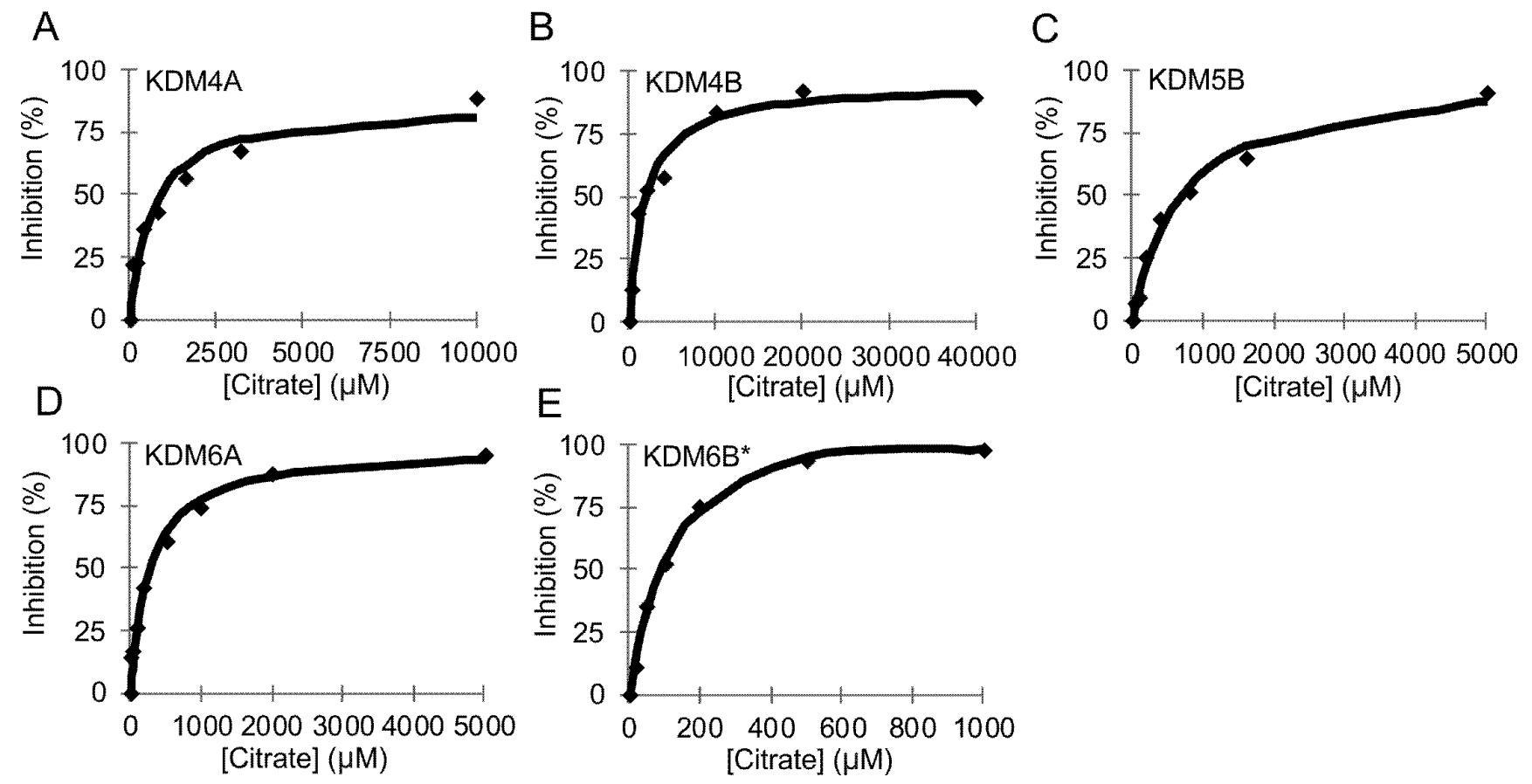

Supp. Fig. 8. KDMs are susceptible to competitive inhibition by citrate. $\mathrm{IC}_{50}$ curves of KDM4A (a), KDM4B (b), KDM5B (c), KDM6A (d) and KDM6B* (e) for citrate. *JmjC domain. 

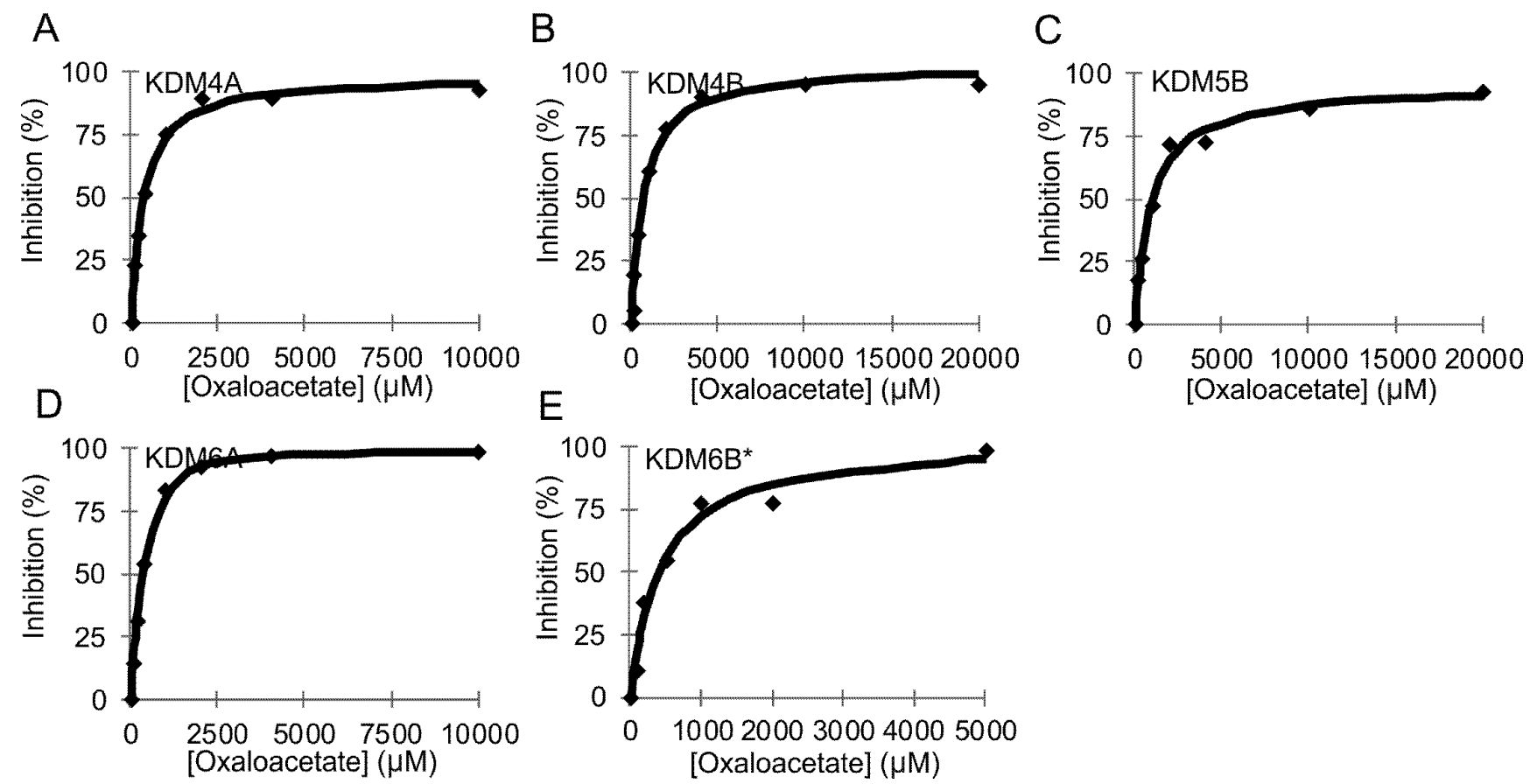

Supp. Fig. 9. KDMs are susceptible to competitive inhibition by oxaloacetate. $\mathrm{IC}_{50}$ curves of KDM4A (a), KDM4B (b), KDM5B (c), KDM6A (d) and KDM6B* (e) for oxaloacetate. *JmjC domain. 


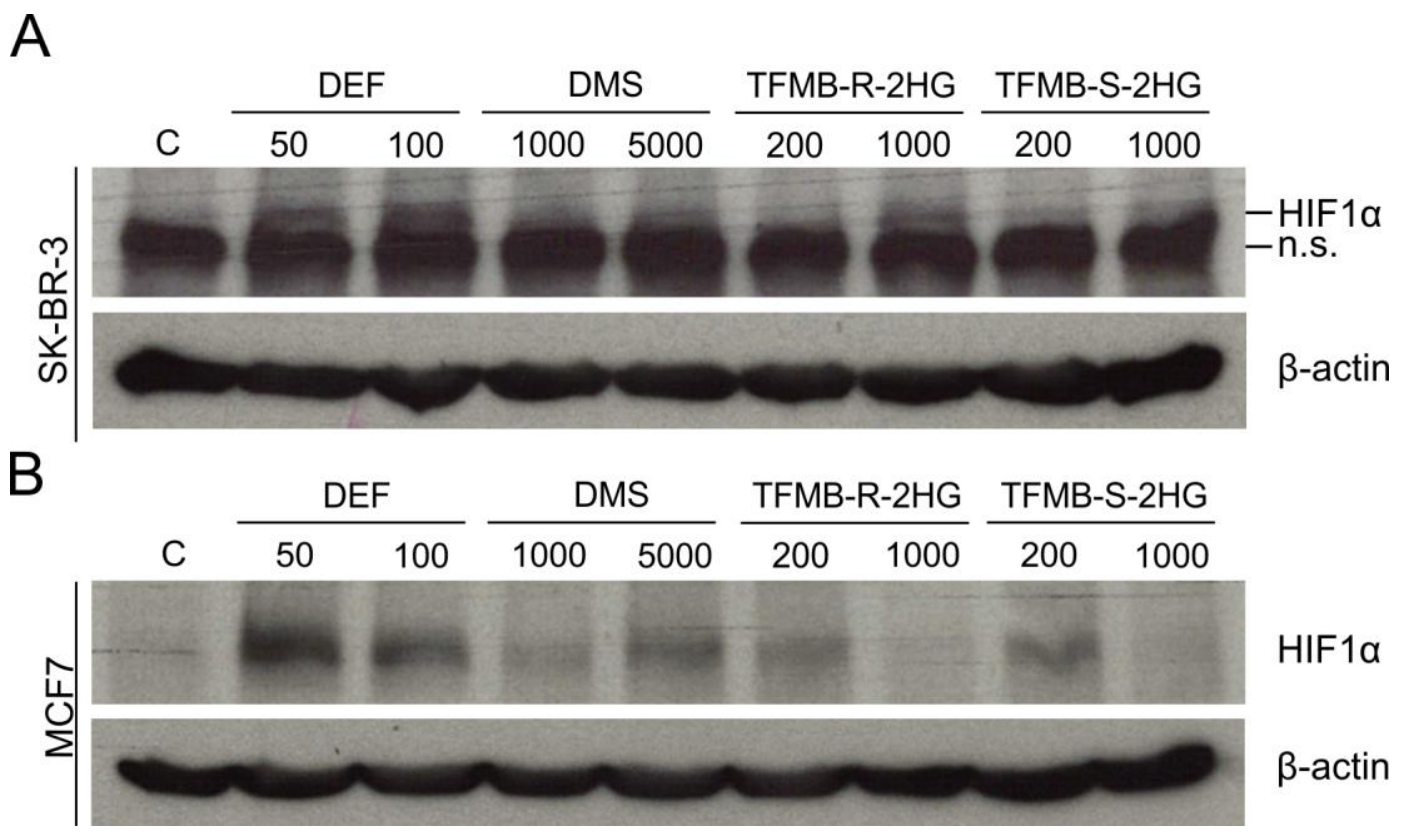

Supp. Fig. 10. Treatment of cells with cell-permeable forms of fumarate, succinate, R-2HG and S-2HG resulted in HIF $1 \alpha$ stabilisation. HIF1 $\alpha$ levels determined by immunoblotting of SK-BR-3 (a) and MCF7 (b) cells incubated with increasing concentrations of diethyl fumarate (DEF), dimethyl succinate (DMS), TFMB-R-2HG and TFMB-S-2HG for 24 or $72 \mathrm{~h}$, respectively. $\beta$-actin was used as a loading control. 